\title{
LAURENT POLYNOMIAL LANDAU-GINZBURG MODELS FOR COMINUSCULE HOMOGENEOUS SPACES
}

\author{
PETER SPACEK \\ SMSAS, University of Kent \\ Canterbury, UK \\ ps508@kent.ac.uk
}

\begin{abstract}
In this article we construct Laurent polynomial Landau-Ginzburg models for cominuscule homogeneous spaces. These Laurent polynomial potentials are defined on a particular algebraic torus inside the Lie-theoretic mirror model constructed for arbitrary homogeneous spaces in [Rie08]. The Laurent polynomial takes a similar shape to the one given in [Giv96] for projective complete intersections, i.e., it is the sum of the toric coordinates plus a quantum term. We also give a general enumeration method for the summands in the quantum term of the potential in terms of the quiver introduced in [CMP08], associated to the Langlands dual homogeneous space. This enumeration method generalizes the use of Young diagrams for Grassmannians and Lagrangian Grassmannians and can be defined type-independently. The obtained Laurent polynomials coincide with the results obtained so far in [PRW16] and [PR13] for quadrics and Lagrangian Grassmannians. We also obtain new Laurent polynomial Landau-Ginzburg models for orthogonal Grassmannians, the Cayley plane and the Freudenthal variety.
\end{abstract}

\section{Contents}

\section{Introduction}

2. Conventions and notation

3. Rietsch's Lie-theoretic mirror model

4. Cominuscule homogeneous spaces and minuscule representations

5. Statement of the Laurent polynomial potential

6. Proof of the Laurent polynomial expression

7. Proof of the intermediate results

8. Reformulating the quantum term using quiver subsets

9. Laurent polynomial potentials for all the cominuscule cases

DOI: $10.1007 / \mathrm{s} 00031-020-09636-7$

Received February 19, 2020. Accepted September 30, 2020.

Published online January 27, 2021.

Corresponding Author: Peter Spacek, e-mail: ps508@kent.ac.uk 


\section{Introduction}

Consider an arbitrary complete homogeneous space $X=G / P$ for a simple and simply-connected complex algebraic group $G$. In [Rie08], Rietsch gives a general construction of a Landau-Ginzburg model that recovers Peterson's presentation of the small quantum cohomology $q H^{*}(X)$ in [Pet97]. In the subsequent articles [MR13], [PR18], [PRW16], [PR13] this general construction is worked out in special cases of cominuscule homogeneous spaces (namely Grassmannians, odd quadrics, all quadrics, and Lagrangian Grassmannians, respectively) to formulate the potentials of these Landau-Ginzburg models in projective coordinates. To obtain these potentials, the articles first formulated Laurent polynomial expressions for these on an algebraic torus.

On comparing the methods used to determine these Laurent polynomial potentials, a general, type-independent method has emerged, which is what we will describe here. This is achieved by modifying the method as used in [PR18] in order to circumvent considerations that only hold in the case of odd quadrics. In particular, we rely on the general structure of minuscule representations as described by the article [Gre08].

We obtain the following expression for the Laurent polynomial potential:

$$
\sum_{i=1}^{\ell} a_{i}+q \frac{\sum_{\left(i_{j}\right) \in \mathcal{I}} a_{i_{1}} \cdots a_{i_{\ell^{\prime}}}}{\prod_{i=1}^{\ell} a_{i}}
$$

where $\ell=\operatorname{dim}(X)$, the $a_{i}$ are the toric coordinates for $i \in\{1, \ldots, \ell\}$, and $q$ is the quantum parameter. The set $\mathcal{I}$ is the set of subexpressions of a certain Weyl group element $w^{\prime}$ in a fixed reduced expression of the minimal coset representative $w^{P}$ of the longest Weyl group element, see equation (8) for $w^{P}$, equation (13) for $w^{\prime}$ and Definition 5.5 for $\mathcal{I}$. For the full statement of the result, see Theorem 5.7.

Notice that the expression in (1) is reminiscent of the Laurent polynomial for projective complete intersections given in [Giv96]. Indeed, it is given as the sum of the toric coordinates $a_{i}$ plus a quantum term consisting of a homogeneous polynomial divided by the product of all the toric coordinates. We give a second type-independent description for this homogeneous polynomial: we replace the summation over $\mathcal{I}$ by a summation over the set $\mathcal{S}$ of special subsets of the quiver $Q_{X}$ associated to $w^{P}$ by [Per07], [CMP08], see Definitions 8.3 and 8.5 as well as Corollary 8.12. These subsets of $Q_{X}$ can be considered as generalizations of Young tableaux used in a similar way.

We use the second type-independent expression to obtain Laurent polynomial potentials for all cominuscule homogeneous spaces: Grassmannians, quadrics, Lagrangian Grassmannians, orthogonal Grassmannians, the Cayley plane, and the Freudenthal variety. The obtained expressions for quadrics and Lagrangian Grassmannians coincide with those given earlier in [PRW16] and [PR13]. This is to be expected from the fact that the type-independent expression is a generalization of these cases. However, to the best of our knowledge, the expressions for orthogonal Grassmannians (for general $n$ ), the Cayley plane and the Freudenthal variety are new. 
The Laurent polynomial potentials will facilitate finding expressions using projective coordinates for the Landau-Ginzburg models constructed in [Rie08] analogously to [MR13], [PR13], [PRW16], [PR18], and this might even be done typeindependently. Furthermore, in [Rie08], Rietsch also conjectures that LandauGinzburg models she constructed give rise to oscillatory integrals that are solutions to the quantum differential equations of $X$, see Conjecture 8.1 there. This conjecture is verified in [PRW16] using their Laurent polynomial expression to describe a flat section of the Dubrovin connection. Thus, we expect that progress can be made in resolving this conjecture for other cominuscule examples using the results obtained here.

The outline of this article is as follows. We start in Section 2 with recalling some of the fundamentals required and fixing notation. This is followed by a short presentation of the results of [Rie08] in Section 3. In Section 4 we restrict to cominuscule homogeneous spaces and consider the general structure of minuscule representations. Next, in Section 5 we state our Laurent polynomial expression (Theorem 5.7) for the potential restricted to an open dense subset. We prove this expression in Section 6, postponing the proof of a number of intermediate results to Section 7 . We deduce an alternative description of the quantum term (Corollary 8.12) using subsets of a specific quiver in Section 8, which simplifies the calculation of the Laurent polynomials. Finally, we apply the expression in Corollary 8.12 to all the cominuscule homogeneous spaces in section 9, verifying that the expression coincides with [PRW16] and [PR13] for quadrics and Lagrangian Grassmannians, and obtaining new Laurent polynomial potentials for orthogonal Grassmannians (Subsection 9.4), the Cayley plane (Subsection 9.5) and the Freudenthal variety (Subsection 9.6). We refer the reader interested in representative examples of the sets $\mathcal{S}$ and the resulting Laurent polynomial expressions to the arXiv version of this paper [Spa19].

Acknowledgments. We would like to thank Dr. C.M.A. Pech for the many helpful discussions and her improvements to the readability of this text.

\section{Conventions and notation}

Let $X$ be a complete homogeneous space (also known as a generalized flag variety) for a simple and simply-connected complex algebraic group $G$ of rank $n$. In this section and in Section 3 we do not make any further assumptions on $X$, but in the remaining sections will specialize to the case in which $X$ is cominuscule, see Section 4.

Write $\mathfrak{g}$ for the Lie algebra of $G$ and fix a set $\left(e_{1}, f_{1}, h_{1}, \ldots, e_{n}, f_{n}, h_{n}\right)$ of Chevalley generators, where $h_{i}=\left[e_{i}, f_{i}\right]$ for $i \in\{1, \ldots, n\}$. This gives the decomposition $\mathfrak{g}=\mathfrak{u}_{+} \oplus \mathfrak{t} \oplus \mathfrak{u}_{-}$, where $\mathfrak{u}_{+}$is generated by $\left\{e_{i} \mid 1 \leq i \leq n\right\}, \mathfrak{u}_{-}$is generated by $\left\{f_{i}\right\}$ and the Cartan subalgebra $\mathfrak{t}$ is spanned by $\left\{h_{i}\right\}$. We denote by $\mathcal{U}_{+}$and $\mathcal{U}_{-}$the universal enveloping algebras of $\mathfrak{u}_{+}$and $\mathfrak{u}_{-}$respectively, and we write their completions as $\widehat{\mathcal{U}}_{+}$and $\widehat{\mathcal{U}}_{-}$.

Let $T$ be the maximal torus and $U_{+}$and $U_{-}$be the nilpotent subgroups of $G$ that have $\mathfrak{t}, \mathfrak{u}_{+}$and $\mathfrak{u}_{-}$as Lie algebras, respectively. Note that we can consider $U_{+}$ and $U_{-}$as lying inside $\widehat{\mathcal{U}}_{+}$and $\widehat{\mathcal{U}}_{-}$respectively and that they are generated by the 
one-parameter subgroups

$$
x_{i}(a)=\exp \left(a e_{i}\right) \quad \text { and } \quad y_{i}(a)=\exp \left(a f_{i}\right)
$$

for $i \in\{1, \ldots, n\}$ and $a \in \mathbb{C}$. Here $\exp \left(a e_{i}\right)=1+a e_{i}+\frac{1}{2} a^{2} e_{i}^{2}+\cdots \in \widehat{\mathcal{U}}_{+}$ and $\exp \left(a f_{i}\right) \in \widehat{\mathcal{U}}_{-}$is given analogously. The subgroup $B_{+}=T U_{+}$defines a Borel subgroup, and its opposite is given by $B_{-}=T U_{-}$. They have $\mathfrak{t} \oplus \mathfrak{u}_{+}$and $\mathfrak{t} \oplus \mathfrak{u}_{-}$as respective associated Lie algebras. There is now a unique parabolic subgroup $P$ containing $B_{+}$such that $X=G / P$. The Lie algebra $\mathfrak{p}$ of $P$ satisfies $\mathfrak{u}_{+} \oplus \mathfrak{t} \subset \mathfrak{p} \subset \mathfrak{u}_{+} \oplus \mathfrak{t} \oplus \mathfrak{u}_{-}=\mathfrak{g}$, i.e., it is generated by $e_{i}$ and $h_{i}$ for all $i \in\{1, \ldots, n\}$ and by certain $f_{i}$, but not necessarily all. We will denote by $I_{P} \subset\{1, \ldots, n\}$ the set of indices such that $\mathfrak{p}$ is generated as a Lie algebra as

$$
\mathfrak{p}=\left\langle e_{i}, h_{i}, f_{j} \mid i \in\{1, \ldots, n\}, j \in I_{P}\right\rangle
$$

and its complement is denoted by $I^{P}=\{1, \ldots, n\} \backslash I_{P}$.

We write $\mathcal{X}$ for the lattice of characters $\chi: T \rightarrow \mathbb{C}^{*}$ of the maximal torus (written additively). Within $\mathcal{X}$, we denote the set of roots by $\Phi \subset \mathcal{X}$ and a base of simple roots $\Delta=\left\{\alpha_{1}, \ldots, \alpha_{n}\right\}$ is determined by the Chevalley generators. The associated sets of positive and negative roots are denoted by $\Phi_{+}$and $\Phi_{-}$ respectively. We denote the cocharacter lattice by $\mathcal{X}^{\vee}$, the coroots by $\Phi^{\vee}$ and the simple coroots by $\alpha_{i}^{\vee}: \mathcal{X} \rightarrow \mathbb{C}$.

With a given root system $\Phi$ and character lattice $\mathcal{X}$, there exists a unique group $G^{\vee}$ determined by having as root system the coroots $\Phi^{\vee}$ and as character lattice the cocharacter lattice $\mathcal{X}^{\vee}$ of $G$. The character lattice $\mathcal{X}^{\vee}$ of $G^{\vee}$ also determines a maximal torus $T^{\vee}$ in $G^{\vee}$. The pair $\left(G^{\vee}, T^{\vee}\right)$ is called the Langlands dual pair associated to $(G, T)$; we call $G^{\vee}$ the Langlands dual group.

Remark 2.1. As $G$ is assumed to be simply-connected, $G^{\vee}$ will be adjoint.

The Langlands dual group $G^{\vee}$ inherits the base $\Delta^{\vee}=\left\{\alpha_{1}^{\vee}, \ldots, \alpha_{n}^{\vee}\right\}$ of simple roots, which in turn determines the decomposition of the Lie algebra $\mathfrak{g}^{\vee}$ of $G^{\vee}$ into $\mathfrak{g}^{\vee}=\mathfrak{u}_{-}^{\vee} \oplus \mathfrak{t}^{\vee} \oplus \mathfrak{u}_{+}^{\vee}$. The Langlands dual groups $U_{+}^{\vee}, U_{-}^{\vee}, B_{+}^{\vee}, B_{-}^{\vee}$ and $P^{\vee}$ are now defined analogously to above, and we write $\Phi_{+}^{\vee}$ and $\Phi_{-}^{\vee}$ for the sets positive and negative roots of $G^{\vee}$. We also obtain Chevalley generators $\left(e_{1}^{\vee}, f_{1}^{\vee}, h_{1}^{\vee}, \ldots\right.$ $\left.\ldots, e_{n}^{\vee}, f_{n}^{\vee}, h_{n}^{\vee}\right)$ and define the corresponding one-parameter subgroups of $U_{+}^{\vee}$ and $U_{-}^{\vee}$

$$
x_{i}^{\vee}(a)=\exp \left(a e_{i}^{\vee}\right) \quad \text { and } \quad y_{i}^{\vee}(a)=\exp \left(a f_{i}^{\vee}\right)
$$

for $i \in\{1, \ldots, n\}$ and $a \in \mathbb{C}$. Here $\exp \left(a e_{i}^{\vee}\right)=1+a e_{i}^{\vee}+\frac{1}{2} a^{2}\left(e_{i}^{\vee}\right)^{2}+\cdots \in \widehat{\mathcal{U}}_{+}^{\vee}$ in the completed universal enveloping algebra of $\mathfrak{u}_{+}^{\vee}$, and analogously for $\exp \left(a f_{i}^{\vee}\right)$. Note that the parabolic subgroup $P^{\vee}$ is associated to the same set $I_{P}$ as $P$ : that is, its Lie algebra is given by

$$
\mathfrak{p}^{\vee}=\left\langle e_{i}^{\vee}, h_{i}^{\vee}, f_{j}^{\vee} \mid i \in\{1, \ldots, n\}, j \in I_{P}\right\rangle .
$$

Thus, the complement of the set of indices is the same, so this is denoted by $I^{P}=\{1, \ldots, n\} \backslash I_{P}$ as well. 
Remark 2.2. When the Dynkin diagram of $G$ is simply-laced, $G^{\vee}$ has the same Dynkin diagram (with the same numbering of the vertices). When the Dynkin diagram of $G$ has a double or triple edge, the Dynkin diagram of $G^{\vee}$ is obtained by reversing the arrows at these edges. Explicitly, if $G$ is of type $\mathrm{A}_{n}, \mathrm{D}_{n}$ or $\mathrm{E}_{n}$, then $G^{\vee}$ is of the same type; if $G$ is of type $\mathrm{B}_{n}$, then $G^{\vee}$ is of type $\mathrm{C}_{n}$ and vice versa; finally, for $G$ of type $\mathrm{F}_{4}$ and $\mathrm{G}_{2}, G^{\vee}$ is of the same type but has the reverse numbering of vertices.

Apart from the Chevalley generators $\left(e_{1}^{\vee}, f_{1}^{\vee}, h_{1}^{\vee}, \ldots, e_{n}^{\vee}, f_{n}^{\vee}, h_{n}^{\vee}\right)$ for $\mathfrak{g}^{\vee}$, we will need the corresponding dual maps $\left(e_{i}^{\vee}\right)^{*},\left(f_{i}^{\vee}\right)^{*} \in\left(\mathfrak{g}^{\vee}\right)^{*}$ as well, satisfying

$$
\left(e_{i}^{\vee}\right)^{*}\left(e_{j}^{\vee}\right)=\delta_{i j}=\left(f_{i}^{\vee}\right)^{*}\left(f_{j}^{\vee}\right) \text { and }\left(e_{i}^{\vee}\right)^{*}\left(f_{j}^{\vee}\right)=0=\left(f_{i}^{\vee}\right)^{*}\left(e_{j}^{\vee}\right)
$$

and vanishing on $\mathfrak{t}^{\vee}$ and the other root spaces of $\mathfrak{g}^{\vee}$.

We extend these maps to be defined on arbitrary products of the Chevalley generators using the inclusions of $\mathfrak{u}_{+}^{\vee}$ and $\mathfrak{u}_{-}^{\vee}$ into their completed universal algebras $\widehat{\mathcal{U}}_{+}^{\vee}$ and $\widehat{\mathcal{U}}_{-}^{\vee}$. This in turn allows us to define $\left(e_{i}^{\vee}\right)^{*}$ and $\left(f_{i}^{\vee}\right)^{*}$ on $U_{+}^{\vee}$ and $U_{-}^{\vee}$ through the identification of the one-parameter subgroups $x_{i}^{\vee}(a) \in U_{+}^{\vee}$ and $y_{i}^{\vee}(a) \in U_{-}^{\vee}$ with $\exp \left(a e_{i}^{\vee}\right) \in \widehat{\mathcal{U}}_{+}^{\vee}$ and $\exp \left(a f_{i}^{\vee}\right) \in \widehat{\mathcal{U}}_{-}^{\vee}$ respectively. Equivalently, $\left(e_{i}^{\vee}\right)^{*}$ and $\left(f_{i}^{\vee}\right)^{*}$ are defined as the unique group homomorphisms $U_{+}^{\vee} \rightarrow \mathbb{C}$ and $U_{-}^{\vee} \rightarrow \mathbb{C}$ such that

$$
\left(e_{i}^{\vee}\right)^{*}\left(x_{j}^{\vee}(a)\right)=a \delta_{i j}=\left(f_{i}^{\vee}\right)^{*}\left(y_{j}^{\vee}(a)\right) .
$$

As $G^{\vee}$ is in general not simply-connected, we will need to consider the universal cover $\widetilde{G}^{\vee}$ of $G^{\vee}$ in Section 6 . As before, we define the universal covers $\widetilde{P}^{\vee}, \widetilde{T}^{\vee}$, $\widetilde{B}_{+}^{\vee}$ and $\widetilde{B}_{-}^{\vee}$. Note that the cover of $U_{+}^{\vee}$ is in fact isomorphic to $U_{+}^{\vee}$ and the same holds for $U_{-}^{\vee}$, so we simply identify them.

Remark 2.3. Considering Remark 2.2, we note that for a simply-connected group $G$ with a simply-laced Dynkin diagram we have $\widetilde{G}^{\vee} \cong G$ as they are both simplyconnected and of the same type.

We turn to the Weyl groups of $G^{\vee}$ and $P^{\vee}$. We denote by $W$ the Weyl group ${ }^{1}$ of $G^{\vee}$, that is the Weyl group associated to the Dynkin diagram of $G^{\vee}$. The Weyl group is generated by the simple reflections denoted by $s_{i}=s_{\alpha_{i}^{\vee}}$ for $\alpha_{i}^{\vee} \in \Delta^{\vee}$ and any expression for $w \in W$ of the form $w=s_{i_{1}} \cdots s_{i_{j}}$ with $j$ minimal is called a reduced expression; in this case the integer $j$ is called the length of $w$ and denoted by $\ell(w)=j$. The longest element of $W$ is denoted by $w_{0}$. We obtain the Weyl group of $P^{\vee}$, denoted by $W_{P}$, by removing the simple reflections $\left\{s_{i} \mid i \in I^{P}\right\}$ from the generators of $W$, compare equation (4). Note that $W_{P}$ is a Weyl group in its own right, associated to the Dynkin diagram of $G^{\vee}$ with the vertices marked by $I^{P}$ removed. The longest element of $W_{P}$ is denoted by $w_{P}$. To each $s_{i} \in W$, we associate two elements in $G^{\vee}$ :

$$
\dot{s}_{i}=x_{i}^{\vee}(1) y_{i}^{\vee}(-1) x_{i}^{\vee}(1) \quad \text { and } \quad \bar{s}_{i}=x_{i}^{\vee}(-1) y_{i}^{\vee}(1) x_{i}^{\vee}(-1)=\dot{s}_{i}^{-1},
$$

${ }^{1}$ Note that the Weyl groups of $G^{\vee}$ and $G$ are isomorphic, as they only depend on the underlying Coxeter diagram of the Dynkin diagrams and these are invariant under Langlands duality. We therefore omit the " $\vee$ " from notation. The same holds for the Weyl group $W_{P}$ of $P^{\vee}$. 
and we extend this to an arbitrary $w \in W$ with reduced expression $w=s_{i_{1}} \cdots s_{i_{d}}$ by setting $\dot{w}=\dot{s}_{i_{1}} \cdots \dot{s}_{i_{d}}$ and $\bar{w}=\bar{s}_{i_{1}} \cdots \bar{s}_{i_{d}}$. Note that $\bar{w}$ is not equal to $\dot{w}^{-1}$ in general: it has the reverse product of simple reflections, i.e., $\dot{w}^{-1}=\bar{s}_{i_{d}} \cdots \bar{s}_{i_{1}}$. Moreover, note that $\dot{s}_{i}$ and $\bar{s}_{i}$ only differ by a torus element and both normalize $T$, so that they indeed map to the same element $s_{i}$ under the identification of $W$ with $N_{G^{\vee}}\left(T^{\vee}\right) / T^{\vee}$, the quotient of the normalizer of the maximal torus $T^{\vee}$ in $G^{\vee}$ by the torus.

We let $T^{P}=\left(T^{\vee}\right)^{W_{P}} \subset T^{\vee}$ be the part of $T^{\vee}$ that is invariant under the action $W_{P} \times T^{\vee} \rightarrow T^{\vee}$ given by $(w, t) \mapsto \dot{w} t \dot{w}^{-1}$. Clearly, $T^{P}$ has dimension $\# I^{P}$.

We denote by $W^{P} \subset W$ the set of minimal coset representatives of $W / W_{P}$ (i.e., for every coset the representative of minimal length), and we denote the minimal representative of $w_{0} W_{P}$ by $w^{P}$. Note that $w_{0}=w^{P} w_{P}$, and we fix reduced expressions

$$
w^{P}=s_{r_{1}} \cdots s_{r_{\ell}} \quad \text { and } \quad w_{P}=s_{q_{1}} \cdots s_{q_{m}},
$$

so we obtain a reduced expression $w_{0}=s_{r_{1}} \cdots s_{r_{\ell}} s_{q_{1}} \cdots s_{q_{m}}$.

\section{Rietsch's Lie-theoretic mirror model}

In [Rie08], Rietsch constructs a Landau-Ginzburg model for general homogeneous spaces $X=G / P$ for $P$ an arbitrary parabolic subgroup. The mirror variety there is a subvariety of the open Richardson variety associated to $\left(w_{P}, w_{0}\right)$. This open Richardson variety is given by:

$$
X^{\vee}=\mathcal{R}_{w_{P}, w_{0}}^{\vee}=\left(B_{+}^{\vee} w_{P} B_{-}^{\vee} \cap B_{-}^{\vee} w_{0} B_{-}^{\vee}\right) / B_{-}^{\vee} \subset G^{\vee} / B_{-}^{\vee},
$$

see [Rie08, Sect. 2]. This variety turns out to be related to the following subset of $G^{\vee}$ :

$$
\mathcal{Z}_{P}^{\vee}=B_{-}^{\vee} \bar{w}_{0}^{-1} \cap U_{+}^{\vee} T^{P} \bar{w}_{P} U_{-}^{\vee} \subset G^{\vee}
$$

Namely, there exists an isomorphism

$$
\Psi: X^{\vee} \times T^{P} \stackrel{\sim}{\longrightarrow} \mathcal{Z}_{P}^{\vee},
$$

whose inverse is given by $z \mapsto\left(z B_{-}^{\vee}, t\right)$ where $z=u_{+} t \bar{w}_{P} u_{-}$.

Remark 3.1. These results are analogous to the statements in [Rie08, Sect. 4.1], although we have modified the definition of $\mathcal{Z}_{P}^{\vee}$ compared to [Rie08] to facilitate calculations in Section 7. Indeed, $\Psi$ can be obtained as follows: For a given $t \in$ $T^{P}$, a class $r B_{-}^{\vee} \in \mathcal{R}_{w_{P}, w_{0}}^{\vee}$ allows by definition a representative of the form $r=$ $b_{-} \bar{w}_{0}^{-1} b_{-}^{\prime}=b_{+} \bar{w}_{P} t u_{-}$(where $b_{-}, b_{-}^{\prime} \in B_{-}^{\vee}, b_{+} \in B_{+}^{\vee}$ and $\left.u_{-} \in U_{-}^{\vee}\right)$. Note that this representative is unique up to right-multiplication with an element of $U_{-}^{\vee}$. Thus, writing $b_{-}^{\prime}=t^{\prime} u_{-}^{\prime}$ for $t^{\prime} \in T^{\vee}$ and $u_{-}^{\prime} \in U_{-}^{\vee}$, the element $r\left(u_{-}^{\prime}\right)^{-1}$ is independent of the choice of representative. Moreover, it is an element of $B_{-}^{\vee} \bar{w}_{0}^{-1}$, as $b_{-} \bar{w}_{0}^{-1} t^{\prime}=b_{-} t^{\prime \prime} \bar{w}_{0}^{-1}$ for some $t^{\prime \prime} \in T^{\vee}$. Writing $b_{+}=t_{+} u_{+}$for $t_{+} \in T^{\vee}$ and $u_{+} \in U_{+}^{\vee}$, we find that $\Psi(z)=t_{+}^{-1} r\left(u_{-}^{\prime}\right)^{-1}$ is well defined and an element of $\mathcal{Z}_{P}^{\vee}$ (using $\bar{w}_{P} t=t \bar{w}_{P}$ ). 
Now, in [Pet97], Peterson gave a presentation of the quantum cohomology of a generalized flag variety $G / P$ as the coordinate ring of what is subsequently called the Peterson variety (see, e.g., [Rie08, par. 3.2]). The coordinate ring of a wellchosen open stratum of this (non-reduced) variety gives the quantum cohomology localized at the quantum parameters (see, e.g., [Rie08, eqn. (3.2)]). In [Rie08], this open stratum is then shown to be isomorphic to the critical locus of a certain function on $\mathcal{Z}_{P}^{\vee}$. Thus, using the isomorphism $\Psi$ of equation (11), we obtain a subvariety of $X^{\vee} \times T^{P}$ whose coordinate ring is also isomorphic to the localized quantum cohomology of $X$.

Instead of presenting these statements in more detail, we will instead follow the reformulation of these results presented in [MR13, Thm. 6.5]; see also [PR18, Sect. 4.2]. There the critical locus is replaced with all of $X^{\vee} \times T^{P}$, but one needs to take the quotient of the coordinate ring by the derivatives with respect to a potential.

Theorem 3.2 ([Rie08, Thm. 4.1], Lie-theoretic LG-model). Let $X=G / P$ be a complete homogeneous space with $G$ a simple, simply-connected algebraic group over $\mathbb{C}$ and with $P$ a (not necessarily maximal) parabolic subgroup. There exists a potential $\mathcal{W}: X^{\vee} \times T^{P} \rightarrow \mathbb{C}$ (given in Definition 3.5$)$ such that

$$
q H^{*}(X)_{\mathrm{loc}} \cong \mathbb{C}\left[X^{\vee} \times T^{P}\right] /\langle\partial \mathcal{W}\rangle,
$$

where $q H^{*}(X)_{\text {loc }}$ is the (small) quantum cohomology of $X$ with all quantum parameters inverted and where $\langle\partial \mathcal{W}\rangle$ is the ideal generated by the derivatives of $\mathcal{W}$ along $X^{\vee}$.

The potential $\mathcal{W}$ is presented in [PR18] as the pull-back of a potential defined on $\mathcal{Z}_{P}^{\vee}$ along the isomorphism $\Psi: X^{\vee} \times T^{P} \stackrel{\sim}{\longrightarrow} \mathcal{Z}_{P}^{\vee}$ from equation (11). To state this potential, we introduce to the following subset of $U_{-}^{\vee}$ :

$$
U_{-}^{P}=U_{-}^{\vee} \cap B_{+}^{\vee} \bar{w}_{P} \bar{w}_{0} B_{+}^{\vee} \subset U_{-}^{\vee}
$$

This set has the following property, which will also be important in Section 5:

Lemma 3.3 ([PR18, Prop. 5.1]). Every $z \in \mathcal{Z}_{P}^{\vee}$ has a unique decomposition $z=$ $u_{+} t \bar{w}_{P} u_{-}$with $u_{+} \in U_{+}^{\vee}, t \in T^{P}$ and $u_{-} \in U_{-}^{P}$. In particular, fixing $\left(u_{-}, t\right)$ determines $u_{+}$.

Remark 3.4. The proof of this result in [PR18, Prop. 5.1] can be carried over to the general case without any modification, so it will be omitted here. Note that our definition of $U_{-}^{P}$ coincides with the one used in equation (7) of [PR18] as $\left(w^{P}\right)^{-1}=w_{P} w_{0}$ and the chosen representatives only differ by a torus element, so that $B_{+}^{\vee} \bar{w}_{P} \bar{w}_{0} B_{+}^{\vee}=B_{+}^{\vee}\left(\dot{w}^{P}\right)^{-1} B_{+}^{\vee}$.

The potential on $\mathcal{Z}_{P}^{\vee}$ is now defined as follows:

Definition 3.5. Define the potential $\mathcal{W}_{\mathcal{Z}_{P}^{\vee}}: \mathcal{Z}_{P}^{\vee} \rightarrow \mathbb{C}$ as the map:

$$
\mathcal{W}_{\mathcal{Z}_{P}^{\vee}}: \quad z=u_{+} t \bar{w}_{P} u_{-} \mapsto \mathcal{E}^{*}\left(u_{+}^{-1}\right)+\mathcal{F}^{*}\left(u_{-}\right)
$$


where $\mathcal{E}^{*}=\sum_{i=1}^{n}\left(e_{i}^{\vee}\right)^{*}$ and $\mathcal{F}^{*}=\sum_{i=1}^{n}\left(f_{i}^{\vee}\right)^{*}$, and where the decomposition of $z=u_{+} t \bar{w}_{P} u_{-}$is the unique decomposition with $u_{-} \in U_{-}^{P}$ as stated in Lemma 3.3. Moreover, the potential $\mathcal{W}: X^{\vee} \times T^{P} \rightarrow \mathbb{C}$ mentioned in Theorem 3.2 is given by $\mathcal{W}=\mathcal{W}_{\mathcal{Z}_{P}} \circ \Psi$ with $\Psi$ given in equation (11).

\section{Cominuscule homogeneous spaces and minuscule representations}

In Section 5 we will give a Laurent polynomial expression for the potential $\mathcal{W}_{\mathcal{Z}_{P}^{\vee}}$ restricted to an open algebraic torus inside $\mathcal{Z}_{P}^{\vee}$, after assuming the homogeneous space is cominuscule. In this section we will discuss this property, fix further notation and finally consider minuscule representations of a given Lie algebra.

We will maintain all the assumptions and conventions of Section 2. In particular, we assume that $X=G / P$ is a homogeneous space for a complex algebraic group $G$ of rank $n$ that is both simple and simply-connected. Now, we assume in addition that $X$ is minimal. This is equivalent to the assumption that $P$ is a maximal parabolic subgroup; that is, $I^{P}=\{k\}$ for a single index $k \in\{1, \ldots, n\}$, see equation (2). Thus, $P$ is associated to a single vertex $k$ of the Dynkin diagram of $G$. This fact is denoted by $P=P_{k}$. Note that the Langlands dual group $P^{\vee}$ is associated to the $k$ th vertex of the Dynkin diagram of $G^{\vee}$ (see equation (4) and Remark 2.2), so we can also write $P^{\vee}=P_{k}^{\vee}$.

Because of the maximality of $P^{\vee}=P_{k}^{\vee}$, we know that $W_{P}=\left\langle s_{i} \mid i \neq k\right\rangle$. In particular, the invariant torus $T^{P} \subset T^{\vee}$ is one-dimensional and $\alpha_{k}^{\vee}: T^{P} \rightarrow$ $\mathbb{C}^{*}$ gives an isomorphism $\left(G^{\vee}\right.$ being adjoint). The element $w_{P} s_{k} \in W$ will turn out to be of particular interest; we will write $w^{\prime \prime} \in W^{P}$ for the minimal coset representative of $w_{P} s_{k} W_{P}$ and denote its length by $\ell\left(w^{\prime \prime}\right)=\ell^{\prime \prime} \leq \ell=\ell\left(w^{P}\right)$. Define $w^{\prime} \in W$ by

$$
w^{\prime}=w^{P}\left(w^{\prime \prime}\right)^{-1}
$$

and write $\ell\left(w^{\prime}\right)=\ell^{\prime}$; clearly $\ell=\ell^{\prime}+\ell^{\prime \prime}$.

The second assumption we will impose on $X=G / P_{k}$ is that it is a cominuscule homogeneous space. A minimal homogeneous space $X=G / P_{k}$ is called (co)minuscule if the fundamental weight $\omega_{k}$ is (co)minuscule. Recall that the fundamental weights $\left\{\omega_{1}, \ldots, \omega_{n}\right\}$ form a basis of the character lattice $\mathcal{X}$ dual to the simple coroot basis $\Delta^{\vee}=\left\{\alpha_{1}^{\vee}, \ldots, \alpha_{n}^{\vee}\right\}$ of the cocharacter lattice $\mathcal{X}^{\vee}$. A fundamental weight $\omega_{i}$ is called minuscule if it satisfies one of the following equivalent conditions (see also [Bou68, Sect. VI.1, exercise 24]):

(i) For every $\alpha^{\vee} \in \Phi^{\vee},\left\langle\left\langle\omega_{i}, \alpha^{\vee}\right\rangle\right\rangle \in\{-1,0,+1\}$, where $\langle\langle\cdot, \cdot\rangle\rangle: \mathcal{X} \times \mathcal{X}^{\vee} \rightarrow \mathbb{C}$ denotes the dual pairing.

(ii) For $\alpha_{0}^{\vee}$ the longest root of the root system $\Phi^{\vee},\left\langle\left\langle\omega_{i}, \alpha_{0}^{\vee}\right\rangle\right\rangle=1$.

(iii) The coefficient of $\alpha_{i}^{\vee}$ in $\alpha_{0}^{\vee}$ is 1 .

A fundamental weight $\omega_{i}$ is called cominuscule if the corresponding coweight $\omega_{i}^{\vee}$ is minuscule. (Recall that the coweights $\left\{\omega_{1}^{\vee}, \ldots, \omega_{n}^{\vee}\right\}$ form a basis of $\mathcal{X}^{\vee}$ dual to the basis of simple root $\Delta=\left\{\alpha_{1}, \ldots, \alpha_{n}\right\}$ of $\mathcal{X}$.) The list of minuscule and cominuscule fundamental weights is well known; we have included it in Table 1 together with the associated minimal homogeneous spaces $X=G / P_{k}$. 
TABLE 1. Table listing for each type of the fundamental weights that are minuscule, cominuscule or both, the associated homogeneous spaces and their dimensions and indexes. In this table, $Q_{n}$ denotes a quadric of dimension $n ; \operatorname{Gr}(k, n)$ denotes the Grassmannian of $k$-dimensional subspaces in $\mathbb{C}^{n} ; \operatorname{LG}(n, 2 n)$ denotes the Lagrangian Grassmannian of maximal isotropic subspaces with respect to the standard symplectic form; $\mathrm{OG}(n, 2 n)$ and $\mathrm{OG}(n, 2 n+1)$ denote (one of the two isomorphic connected components of) the orthogonal Grassmannians of maximal isotropic subspaces with respect to the standard quadratic form; $\mathbb{O P}^{2}=\mathrm{E}_{6}^{\mathrm{sc}} / P_{6}$ denotes the Cayley plane which is a homogeneous space for $\mathrm{E}_{6}^{\mathrm{sc}}$, the simply-connected Lie group of type $\mathrm{E}_{6}$; and finally $\mathrm{E}_{7}^{\mathrm{sc}} / P_{7}$ is called the Freudenthal variety and is homogeneous for $\mathrm{E}_{7}^{\mathrm{sc}}$, the simply-connected Lie group of type $E_{7}$. Note that the two varieties that are only minuscule are redundant: the type- $\mathrm{B}_{n}$ minuscule variety $\mathrm{OG}(n, 2 n+1)$ is isomorphic to the variety $\mathrm{OG}(n+1,2 n+2)$ which is both minuscule and cominuscule as a type- $\mathrm{D}_{n+1}$ homogeneous space; similarly, the type- $C_{n}$ minuscule variety $\mathbb{C P}^{2 n-1}$ is of course the same as $\operatorname{Gr}(1,2 n)$, which is both minuscule and cominuscule as a type- $\mathrm{A}_{2 n-1}$ homogeneous space. Adapted from [CMP08].

\begin{tabular}{|c|c|c|c|c|c|c|}
\hline \multicolumn{4}{|c|}{ type and (co)minuscule weight } & variety & $\operatorname{dim}$ & index \\
\hline $\mathrm{A}_{n-1}$ & $\bullet \bullet \cdots \longrightarrow$ & any $k$ & both & $\operatorname{Gr}(k, n)$ & $k(n-k)$ & $n$ \\
\hline $\mathrm{B}_{n}$ & $\bullet-\cdots-\cdots>0$ & 1 & com. & $Q_{2 n-1}$ & $2 n-1$ & $2 n-1$ \\
\hline $\mathrm{B}_{n}$ & $\circ-\cdots \multimap \Longrightarrow$ & $n$ & $\min$. & $\mathrm{OG}(n, 2 n+1)$ & $\frac{1}{2} n(n+1)$ & $2 n$ \\
\hline $\mathrm{C}_{n}$ & - $-0-\cdots-0<0$ & 1 & $\min$. & $\mathbb{C} P^{2 n-1}$ & $2 n-1$ & $2 n$ \\
\hline $\mathrm{C}_{n}$ & $0-\cdots-\cdots$ & $n$ & com. & $\mathrm{LG}(n, 2 n)$ & $\frac{1}{2} n(n+1)$ & $n+1$ \\
\hline $\mathrm{D}_{n}$ & $\cdot-\cdots-\infty$ & 1 & both & $Q_{2 n-2}$ & $2 n-2$ & $2 n-2$ \\
\hline $\mathrm{D}_{n}$ & $\infty-\cdots-\infty$ & $n-1$ or $n$ & both & $\mathrm{OG}(n, 2 n)$ & $\frac{1}{2} n(n-1)$ & $2 n-2$ \\
\hline $\mathrm{E}_{6}$ & & 1 or 6 & both & $\mathbb{O} \mathbb{P}^{2}=\mathrm{E}_{6}^{\mathrm{sc}} / P_{6}$ & 16 & 12 \\
\hline $\mathrm{E}_{7}$ & 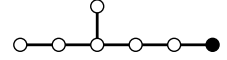 & 7 & both & $\mathrm{E}_{7}^{\mathrm{sc}} / P_{7}$ & 27 & 18 \\
\hline
\end{tabular}

Thus, assuming that $X=G / P_{k}$ is cominuscule means that $\omega_{k}^{\vee}$ is minuscule, which in turn implies that the fundamental weight representation $V_{\omega_{k}^{\vee}}$ is minuscule; that is, the Weyl group acts transitively on the weight spaces of $V_{\omega_{k}}$. Here the simple reflection $s_{i} \in W$ acts on a vector $v_{\mu} \vee$ of weight $\mu^{\vee}$ by mapping it to the vector $\bar{s}_{i} \cdot v_{\mu \vee}$ of weight $s_{i}\left(\mu^{\vee}\right)$.

Recall that the fundamental weight representation $V_{\omega_{k}^{\vee}}$ is the highest weight representation of $\mathfrak{g}^{\vee}$ with $\omega_{k}^{\vee}$ as highest weight. For any choice of a highest-weight vector $v_{\omega_{k}^{\vee}}^{+}$, we obtain the representation as $V_{\omega_{k}^{\vee}}=\mathcal{U}_{-}^{\vee} \cdot \mathbb{C} v_{\omega_{k}^{\vee}}^{+}$, where $\mathcal{U}_{-}^{\vee}$ is the universal enveloping algebra of $\mathfrak{u}_{-}^{\vee}$ (see for example [Hum78, Thm. 20.2]). Thus, $\left\{f_{i_{1}}^{\vee} \cdots f_{i_{j}}^{\vee} \cdot v_{\omega_{k}^{\vee}}^{+} \mid j \geq 0\right\}$ spans $V_{\omega_{k}^{\vee}}$. We want to compare the actions of $\mathcal{U}_{-}^{\vee}$ and $W$, so we will need some results on the structure of minuscule representations. 
Remark 4.1. To be able to apply the results directly to our case, we will change the notation of the following theorem to conform to our situation; for example, we write $\mathfrak{g}^{\vee}$ for a general Lie algebra, as we want to apply the theorem to the minuscule highest weight representation of the Lie algebra $\mathfrak{g}^{\vee}$ of the (adjoint) Langlands dual group $G^{\vee}$ associated to the (simply-connected) group $G$ such that $X=G / P_{k}$.

We denote the Cartan integers of a root system $\Phi^{\vee}$ by $a_{i j} \in \mathbb{Z}, i, j \in\{1, \ldots, n\}$. They are given by $a_{i j}=2\left(\alpha_{j}^{\vee}, \alpha_{i}^{\vee}\right) /\left(\alpha_{i}^{\vee}, \alpha_{i}^{\vee}\right)$ for any choice of non-degenerate, symmetric, bilinear form $(\cdot, \cdot)$ (e.g., the Killing form). We also use the notation $c\left(\mu^{\vee}, \alpha_{i}^{\vee}\right)=2\left(\mu^{\vee}, \alpha_{i}^{\vee}\right) /\left(\alpha_{i}^{\vee}, \alpha_{i}^{\vee}\right)$ for $\mu^{\vee}$ a general weight.

Theorem 4.2 (Green [Gre08]). Let $\mathfrak{g}^{\vee}$ be a simple Lie algebra and fix a set of simple roots $\Delta^{\vee}=\left\{\alpha_{1}^{\vee}, \ldots, \alpha_{n}^{\vee}\right\}$ and Chevalley generators $\left(e_{i}^{\vee}, f_{i}^{\vee}, h_{i}^{\vee}\right)$. Suppose $V\left(\lambda^{\vee}\right)$ is a minuscule representation of $\mathfrak{g}^{\vee}$ with highest weight $\lambda^{\vee}$. Denote by $M\left(\lambda^{\vee}\right)$ the weights of $V\left(\lambda^{\vee}\right)$ and let $\mu^{\vee} \in M\left(\lambda^{\vee}\right)$ be an arbitrary weight. The following statements hold:

(i) $c\left(\mu^{\vee}, \alpha_{i}^{\vee}\right) \in\{-1,0,1\}$, and $\mu^{\vee}-c \alpha_{i}^{\vee} \in M\left(\lambda^{\vee}\right)$ if and only if $c=c\left(\mu^{\vee}, \alpha_{i}^{\vee}\right)$.

(ii) Each of the weight spaces is one-dimensional.

(iii) Given a highest weight vector $v_{\lambda^{\vee}}^{+}$, we can find a basis $\left\{v_{\mu^{\vee}} \mid \mu^{\vee} \in M\left(\lambda^{\vee}\right)\right\}$ with the following properties: $v_{\mu} \vee$ has weight $\mu^{\vee}$ and the basis vector of the highest weight $v_{\lambda^{\vee}}$ coincides with $v_{\lambda \vee}^{+}$; the Chevalley generators act on $v_{\mu} \vee$ as

$$
\begin{aligned}
& e_{i}^{\vee} \cdot v_{\mu^{\vee}}= \begin{cases}v_{\mu^{\vee}+\alpha_{i}^{\vee},} & \text { if } c\left(\mu^{\vee}, \alpha_{i}^{\vee}\right)=-1, \\
0, & \text { otherwise, }\end{cases} \\
& f_{i}^{\vee} \cdot v_{\mu^{\vee}}= \begin{cases}v_{\mu^{\vee}-\alpha_{i}^{\vee},} & \text { if } c\left(\mu^{\vee}, \alpha_{i}^{\vee}\right)=+1, \\
0, & \text { otherwise, }\end{cases}
\end{aligned}
$$

and $h_{i}^{\vee} \cdot v_{\mu^{\vee}}=c\left(\mu^{\vee}, \alpha_{i}\right) v_{\mu^{\vee}}$.

(iv) For any $v \in V\left(\lambda^{\vee}\right)$ and any $i \in\{1, \ldots, n\}$, we have $\left(e_{i}^{\vee}\right)^{2} \cdot v=0$ and $\left(f_{i}^{\vee}\right)^{2} \cdot v=0$. Moreover, if $j \in\{1, \ldots, n\}$ is such that the Cartan integer $a_{i j}=-1$ (i.e., when in the Dynkin diagram we have $i \bullet j$ or $\left.i \gg j\right)^{2}$ we have both $e_{i}^{\vee} e_{j}^{\vee} e_{i}^{\vee} \cdot v=0$ and $f_{i}^{\vee} f_{j}^{\vee} f_{i}^{\vee} \cdot v=0$. Finally, if $a_{i j}<0$ we have $e_{i}^{\vee} f_{j}^{\vee} \cdot v=0=f_{j}^{\vee} e_{i}^{\vee} \cdot v$.

The following corollary is obtained directly by applying Theorem 4.2 to the definition of $\dot{s}_{i}$ and $\bar{s}_{i}$ given in equation (7).

Corollary 4.3. With the assumptions of Theorem 4.2, write $c=c\left(\mu^{\vee}, \alpha_{i}^{\vee}\right)$, then we have $s_{i}\left(\mu^{\vee}\right)=\mu^{\vee}-c \alpha_{i}^{\vee}$ and

$$
\begin{aligned}
& \dot{s}_{i} \cdot v_{\mu^{\vee}}= \begin{cases}v_{\mu^{\vee}+\alpha_{i}^{\vee}}=e_{i}^{\vee} \cdot v_{\mu^{\vee}}, & \text { if } c=-1, \\
v_{\mu^{\vee}}, & \text { if } c=0, \\
-v_{\mu^{\vee}-\alpha_{i}^{\vee}}=-f_{i}^{\vee} \cdot v_{\mu^{\vee}}, & \text { if } c=+1,\end{cases} \\
& \bar{s}_{i} \cdot v_{\mu^{\vee}}= \begin{cases}-v_{\mu^{\vee}+\alpha_{i}^{\vee}}=-e_{i}^{\vee} \cdot v_{\mu^{\vee}}, & \text { if } c=-1, \\
v_{\mu^{\vee}}, & \text { if } c=0, \\
v_{\mu^{\vee}-\alpha_{i}^{\vee}}=f_{i}^{\vee} \cdot v_{\mu^{\vee}}, & \text { if } c=+1,\end{cases}
\end{aligned}
$$

${ }^{2}$ Strictly speaking, we also have $a_{i j}=-1$ when we have $i \Longleftrightarrow j$, but these edges only appear in the Dynkin diagram of type $\mathrm{G}_{2}$, and the corresponding Lie algebra does not have any minuscule representations. 
Conversely, we have

$$
\begin{aligned}
& e_{i}^{\vee} \cdot v_{\mu^{\vee}}= \begin{cases}v_{\mu^{\vee}+\alpha_{i}^{\vee}}=\dot{s}_{i} \cdot v_{\mu^{\vee}}=-\bar{s}_{i} \cdot v_{\mu^{\vee}}, & \text { if } c=-1, \\
0, & \text { otherwise, }\end{cases} \\
& f_{i}^{\vee} \cdot v_{\mu^{\vee}}= \begin{cases}v_{\mu^{\vee}-\alpha_{i}}=\bar{s}_{i} \cdot v_{\mu^{\vee}}=-\dot{s}_{i} \cdot v_{\mu^{\vee}}, & \text { if } c=+1, \\
0, & \text { otherwise. }\end{cases}
\end{aligned}
$$

We now return to the case where $\mathfrak{g}^{\vee}$ is the Lie algebra of the (adjoint) Langlands dual group $G^{\vee}$ of the (simply-connected) Lie group $G$ such that $X=G / P_{k}$ is cominuscule. Thus, the highest weight representation $V_{\omega_{k}^{\vee}}$ of $\mathfrak{g}^{\vee}$ is minuscule and we can apply Corollary 4.3 to $V_{\omega_{k}^{\vee}}$ to obtain the following facts regarding the action of the Weyl group $W$ on the highest weight vector $v_{\omega_{k}^{v}}^{+}$:

Lemma 4.4. Consider the highest weight vector $v_{\omega_{k}^{\vee}}^{+}$of the minuscule fundamental weight representation $V_{\omega_{k}^{\vee}}$, where $k$ is such that $X=G / P_{k}$.

(i) Given an arbitrary $w \in W$ with minimal coset representative $w_{c} \in W^{P}$, then we have $\bar{w} \cdot v_{\omega_{k}^{v}}^{+}=\bar{w}_{c} \cdot v_{\omega_{k}^{v}}^{+}$and $\dot{w} \cdot v_{\omega_{k}^{v}}^{+}=\dot{w}_{c} \cdot v_{\omega_{k}^{v}}^{+}$.

(ii) An element $w_{c} \in W^{P}$ with reduced expression $w_{c}=s_{i_{1}} \cdots s_{i_{c}}$ acts on the vector $v_{\omega_{k}^{\vee}}^{+}$by $\bar{w}_{c} \cdot v_{\omega_{k}^{\vee}}^{+}=f_{i_{1}}^{\vee} \cdots f_{i_{c}}^{\vee} \cdot v_{\omega_{k}^{\vee}}^{+}$and by $\dot{w}_{c} \cdot v_{\omega_{k}^{\vee}}^{+}=(-1)^{c} f_{i_{1}}^{\vee} \cdots f_{i_{c}}^{\vee}$. $v_{\omega_{k}^{v}}^{+}$.

(iii) Conversely, if $f_{i_{1}}^{\vee} \cdots f_{i_{j}}^{\vee} \cdot v_{\omega_{k}^{\vee}}^{+}$is non-zero of weight $\mu^{\vee}$, then $s_{i_{1}} \cdots s_{i_{j}}$ is a reduced expression for the (unique) element $w_{c} \in W^{P}$ such that $w_{c} \cdot \omega_{k}^{\vee}=$ $\mu^{\vee}$.

(iv) In particular, $\bar{w}_{P}, \dot{w}_{P}$ and their inverses act trivially on $v_{\omega_{k}^{v}}^{+}$and we have that the lowest weight vector defined by $v_{\omega_{k}^{\vee}}^{-}=\bar{w}_{0} \cdot v_{\omega_{k}^{\vee}}^{+}$satisfies $v_{\omega_{k}^{\vee}}^{-}=\bar{w}^{P}$. $v_{\omega_{k}^{\vee}}^{+}=f_{r_{1}}^{\vee} \cdots f_{r_{\ell}}^{\vee} \cdot v_{\omega_{k}^{\vee}}^{+}$, where $w^{P}=s_{r_{1}} \cdots s_{r_{\ell}}$ is the reduced expression fixed in equation (8). Moreover, if $f_{i_{1}}^{\vee} \cdots f_{i_{j}}^{\vee} \cdot v_{\omega_{k}^{\vee}}^{+}=v_{\omega_{k}^{\vee}}^{-}$, then $s_{i_{1}} \cdots s_{i_{j}}=w^{P}$ and this is a reduced expression.

Proof. (i) Recall the identity $s_{j}\left(\omega_{k}^{\vee}\right)=\omega_{k}^{\vee}$ for $j \neq k$, i.e., when $s_{j} \in W_{P}$. This implies that an arbitrary $w \in W$ acts on $\omega_{k}^{\vee}$ by its minimal coset representative $w_{c}$ in $W^{P}$. Corollary 4.3 implies that $\bar{w} \cdot v_{\omega_{k}^{\vee}}^{+}=\bar{w}_{c} \cdot v_{\omega_{k}^{\vee}}^{+}$and $\dot{w} \cdot v_{\omega_{k}^{\vee}}^{+}=\dot{w}_{c} \cdot v_{\omega_{k}^{\vee}}^{+}$.

(ii) We need to show that each factor of $\bar{w}_{c}=\bar{s}_{i_{1}} \cdots \bar{s}_{r_{c}}$ acts as $f_{i}^{\vee}$ on $v_{\omega_{k}^{\vee}}^{+}$. Considering Corollary 4.3 , each $\bar{s}_{i}$ acts either by $f_{i}^{\vee},-e_{i}^{\vee}$ or as the identity map. Clearly, none of these factors acts as the identity, as we could remove it from the product, which would contradict the minimality of the coset representative $\dot{w}_{c}$. Moreover, none of the factors acts as $-e_{i}^{\vee}$ either, because of the following argument:

Let $\bar{s}_{i}$ be the right-most factor acting as $-e_{i}^{\vee}$. As $v_{\omega_{k}^{\vee}}^{+}$is the highest weight vector, we have $e_{i}^{\vee} \cdot v_{\omega_{k}^{\vee}}^{+}=0$ (as $e_{i}^{\vee}$ raises the height of the weight), so there must be a number of $\bar{s}_{j}$ in between $\bar{s}_{i}$ and $v_{\omega_{k}^{\vee}}^{+}$acting as $f_{j}^{\vee}$. Let $f_{j}^{\vee}$ be the factor next to $e_{i}^{\vee}$. There are three cases: $j \neq i$ and $a_{i j}<0 ; j \neq i$ and $a_{i j}=0$; and $j=i$. When $j \neq i$ and $a_{i j}<0$, Theorem 4.2(iv) tells us that $e_{i}^{\vee} f_{j}^{\vee} \cdot v=0$ in the representation, 
which is impossible. When $j \neq i$ and $a_{i j}=0$, we know that $e_{i}^{\vee}$ and $f_{j}^{\vee}$ commute in the Lie algebra, so we can assume without loss of generality that only the case $j=i$ occurs. In the case $j=i$ we obtain $e_{i}^{\vee} f_{j}^{\vee} \cdot v=v$ according to Theorem 4.2(iii). However, this is in contradiction with the fact that $w_{c}$ is a minimal coset representative. Thus, all of the factors $\bar{s}_{i}$ of $\bar{w}^{P}$ act as $f_{i}^{\vee}$.

For the equality $\dot{w}_{c}=(-1)^{c} f_{i_{1}}^{\vee} \cdots f_{i_{c}}^{\vee} \cdot v_{\omega_{k}^{\vee}}^{+}$, we use an analogous argument combined with the fact that $\dot{s}_{i}$ acts as either $e_{i}^{\vee},-f_{i}^{\vee}$ or the identity due to Corollary 4.3.

(iii) Let $w=s_{i_{1}} \cdots s_{i_{j}}$ and let $w_{c} \in W^{P}$ denote the minimal length representative of $w W_{P}$. Given a reduced expression $w_{c}=s_{i_{1}^{\prime}} \cdots s_{i_{c}^{\prime}}$, parts (i) and (ii) imply that

$$
\bar{w} \cdot v_{\omega_{k}^{\vee}}^{+}=\bar{w}_{c} \cdot v_{\omega_{k}^{\vee}}^{+}=f_{i_{1}^{\vee}}^{\vee} \cdots f_{i_{c}^{\prime}}^{\vee} \cdot v_{\omega_{k}^{\vee}}^{+} .
$$

Thus, $\bar{w} \cdot v_{\omega_{k}^{\vee}}^{+}$has weight $\omega_{k}^{\vee}-\alpha_{i_{1}^{\prime}}^{\vee}-\cdots-\alpha_{i_{c}^{\prime}}^{\vee}$ by Theorem 4.2(iii).

On the other hand, $f_{i_{1}}^{\vee} \cdots f_{i_{j}}^{\vee} \cdot v_{\omega_{k}^{\vee}}^{+}=\bar{s}_{i_{1}} \cdots \bar{s}_{i_{j}} \cdot v_{\omega_{k}^{\vee}}^{+}=\bar{w} \cdot v_{\omega_{k}^{\vee}}^{+}$by Corollary 4.3, since none of the factors act as the zero map. This has two implications. Firstly, $w=s_{i_{1}} \cdots s_{i_{j}}$ is a reduced expression: else, one of the factors of $f_{i_{1}}^{\vee} \cdots f_{i_{j}}^{\vee}$ must act as the identity map, which contradicts Theorem 4.2(iii). Secondly, the weight of $\bar{w} \cdot v_{\omega_{k}^{\vee}}^{+}$can also be written as $\omega_{k}^{\vee}-\alpha_{i_{1}}^{\vee}-\ldots-\alpha_{i_{j}}^{\vee}$.

We conclude that

$$
\omega_{k}^{\vee}-\alpha_{i_{1}^{\prime}}^{\vee}-\cdots-\alpha_{i_{c}^{\prime}}^{\vee}=w\left(\omega_{k}^{\vee}\right)=\omega_{k}^{\vee}-\alpha_{i_{1}}^{\vee}-\ldots-\alpha_{i_{j}}^{\vee} .
$$

Clearly, this can only hold when $c=\ell\left(w_{c}\right)=\ell(w)=j$.

Thus, we have $w=w_{c} \in W^{P}$ and we have already shown that $s_{i_{1}} \cdots s_{i_{j}}$ is a reduced expression for $w$. Moreover, $w\left(\omega_{k}^{\vee}\right)=\mu^{\vee}$ by definition and this determines $w$ uniquely, proving (iii).

(iv) As $w_{P} \in W_{P}$, (i) implies that $\bar{w}_{P}, \dot{w}_{P}$ and their inverses act trivially on $v_{\omega_{k}^{v}}^{+}$. As $w^{P}$ is defined as the minimal coset representative of $w_{0}$, we conclude that $v_{\omega_{k}^{\vee}}^{-}=\bar{w}_{0} \cdot v_{\omega_{k}^{v}}^{+}=\bar{w}^{P} \cdot v_{\omega_{k}^{\vee}}^{+}$by (i), and since $w^{P}=s_{r_{1}} \cdots s_{r_{\ell}}$ is the reduced expression we fixed in Section 2, (ii) implies that $v_{\omega_{k}^{\vee}}^{-}=\bar{s}_{r_{1}} \cdots \bar{s}_{r_{\ell}} \cdot v_{\omega_{k}^{\vee}}^{+}=f_{r_{1}}^{\vee} \cdots f_{r_{\ell}}^{\vee} \cdot v_{\omega_{k}^{\vee}}^{+}$. Part (iii) implies the last statement directly.

\section{Statement of the Laurent polynomial potential}

In this section we state the main result of this article, Theorem 5.7, which is an explicit Laurent polynomial expression for $\mathcal{W}_{\mathcal{Z}_{P}^{\vee}}$ on an open, dense algebraic torus $\mathcal{Z}_{P}^{\circ}$ inside $\mathcal{Z}_{P}^{\vee}$, whenever $X=G / P$ is a cominuscule homogeneous space. Recall that $X=G / P$ is cominuscule when $P=P_{k}$ is maximal and $\omega_{k}^{\vee}$ is minuscule.

First we define another subset of $U_{-}^{\vee}$ :

Definition 5.1. Recall the reduced expression $w^{P}=s_{r_{1}} \cdots s_{r_{\ell}}$ fixed in equation (8). Let $U_{-}^{\circ} \subset U_{-}^{\vee}$ be the algebraic torus of elements $u_{-}$that can be written as

$$
u_{-}=y_{r_{\ell}}^{\vee}\left(a_{\ell}\right) \cdots y_{r_{1}}^{\vee}\left(a_{1}\right)
$$

with $a_{i} \in \mathbb{C}^{*}$. 
Lemma 5.2. We have $U_{-}^{\circ} \subset U_{-}^{P}$ open and dense, where $U_{-}^{P}=U_{-}^{\vee} \cap B_{+}^{\vee} \bar{w}_{P} \bar{w}_{0} B_{+}^{\vee}$ was defined in equation (12).

Proof. (See also [PR18, Sect. 5.2].) Note that

$$
B_{+}^{\vee} \dot{s}_{i} B_{+}^{\vee}=B_{+}^{\vee} x_{i}^{\vee}(1) y_{i}^{\vee}(-1) x_{i}^{\vee}(1) B_{+}^{\vee}=B_{+}^{\vee} y_{i}^{\vee}(-1) B_{+}^{\vee},
$$

so that $y_{i}^{\vee}(a) \in B_{+}^{\vee} \dot{s}_{i} B_{+}^{\vee}$, as the -1 can be scaled to any $a \in \mathbb{C}^{*}$ since $T^{\vee} \subset B_{+}^{\vee}$. Of course, $s_{r_{\ell}} \cdots s_{r_{1}}=\left(w^{P}\right)^{-1}$ is reduced, so the Bruhat lemma (see for example [Hum75, Lem. 29.3.A]) implies that

$$
U_{-}^{\circ} \subset B_{+}^{\vee} \dot{s}_{r_{\ell}} \cdots \dot{s}_{r_{1}} B_{+}^{\vee}=B_{+}^{\vee}\left(\bar{w}^{P}\right)^{-1} B_{+}^{\vee}
$$

and it remains to show that $B_{+}^{\vee}\left(\bar{w}^{P}\right)^{-1} B_{+}^{\vee}=B_{+}^{\vee} \bar{w}_{P} \bar{w}_{0} B_{+}^{\vee}$. This follows from the fact that $w_{0}=w^{P} w_{P}$ and the fact that $\dot{s}_{i}$ and $\bar{s}_{i}$ only differ by a torus element.

It is clear that $U_{-}^{\circ} \subset U_{-}^{P}$ is an open subset and it is dense as both have dimension $\ell\left(w_{P} w_{0}\right)$ and $U_{-}^{P}$ is irreducible [Lus94].

Definition 5.3. We define the open, dense algebraic torus $\mathcal{Z}_{P}^{\circ} \subset \mathcal{Z}_{P}^{\vee}$ as

$$
\mathcal{Z}_{P}^{\circ}=B_{-}^{\vee} \bar{w}_{0}^{-1} \cap U_{+}^{\vee} T^{P} \bar{w}_{P} U_{-}^{\circ} \subset \mathcal{Z}_{P}^{\vee}
$$

Note that the fact that this variety is non-zero and an algebraic torus follows from Lemma 3.3 and the resulting isomorphism $\mathcal{Z}_{P}^{\vee} \rightarrow U_{-}^{P} \times T^{P}: z \mapsto\left(u_{-}, t\right)$.

The following is immediate from the definition and Lemma 3.3:

Corollary 5.4. Every $z \in \mathcal{Z}_{P}^{\circ}$ can be factorized in two ways: on the one hand as $b_{-} \bar{w}_{0}^{-1}$ for $b_{-} \in B_{-}^{\vee}$; and on the other as $z=u_{+} t \bar{w}_{P} u_{-}$with $u_{-} \in U_{-}^{\circ}$ of the form (14), $u_{+} \in U_{+}^{\vee}$ and $t \in T^{P}$. Moreover, the latter decomposition is unique with $u_{+}$ determined by a choice of $\left(u_{-}, t\right)$.

We will define the Laurent polynomial expression for the potential on this algebraic torus $\mathcal{Z}_{P}^{\circ}$. It turns out that this expression is indexed by the subexpressions of $w^{\prime}$ in $w^{P}$. Recall from equation (13) that $w^{\prime} \in W$ is defined by $w^{P}=w^{\prime} w^{\prime \prime}$, where $w^{P}, w^{\prime \prime} \in W^{P}$ are the minimal coset representatives of $w_{0} W_{P}$ and $w_{P} s_{k} W_{P}$ (with $k$ such that $P=P_{k}$ ). Moreover, their lengths are denoted by $\ell\left(w^{P}\right)=\ell$, $\ell\left(w^{\prime}\right)=\ell^{\prime}$ and $\ell\left(w^{\prime \prime}\right)=\ell^{\prime \prime}$ and satisfy $\ell=\ell^{\prime}+\ell^{\prime \prime}$.

Definition 5.5. Let $\mathcal{I}$ be the set indexing reduced subexpressions for $w^{\prime}$ occurring inside the fixed reduced expression $w^{P}=s_{r_{1}} \cdots s_{r_{\ell}}$ of equation (8). In other words:

$$
\mathcal{I}=\left\{\left(i_{1}, \ldots, i_{\ell^{\prime}}\right) \mid 1 \leq i_{1}<i_{2}<\cdots<i_{\ell^{\prime}} \leq \ell \text { and } w^{\prime}=s_{r_{i_{1}}} \cdots s_{r_{i_{\ell^{\prime}}}}\right\}
$$

Remark 5.6. Note that the reduced expression $w^{\prime}=s_{r_{i_{1}}} \cdots s_{r_{i_{\ell^{\prime}}}}$ is not fixed, i.e., if $\left(i_{j}\right),\left(i_{j}^{\prime}\right) \in \mathcal{I}$, then we do not necessarily have $r_{i_{j}}=r_{i_{j}^{\prime}}$ for all $j$. 
Theorem 5.7 (Laurent-polynomial LG-model). Let $X=G / P$ be a cominuscule complete homogeneous space with $G$ a simply-connected, simple, complex algebraic group and $P=P_{k}$ a (maximal) parabolic subgroup. The restriction $\mathcal{W}_{\mathcal{Z}_{P}^{\circ}}$ of $\mathcal{W}_{\mathcal{Z}_{P}}$ to $\mathcal{Z}_{P}^{\circ}$ has the following Laurent polynomial expression:

$$
\mathcal{W}_{\mathcal{Z}_{P}^{\circ}}(z)=\sum_{i=1}^{\ell} a_{i}+q \frac{\sum_{\left(i_{j}\right) \in \mathcal{I}} a_{i_{1}} \cdots a_{i_{\ell^{\prime}}}}{\prod_{i=1}^{\ell} a_{i}} .
$$

Here $z \in \mathcal{Z}_{P}^{\circ}$ is uniquely decomposed as $z=u_{+} t \bar{w}_{P} u_{-}$with $u_{-}=y_{r_{\ell}}^{\vee}\left(a_{\ell}\right) \cdots y_{r_{1}}^{\vee}\left(a_{1}\right)$ in $U_{-}^{\circ}$ as in Corollary 5.4, and $q \in \mathbb{C}^{*}$ is given by $q=\alpha_{k}^{\vee}(t)$ (with $\left.t \in T^{P}\right)$. Finally, the set $\mathcal{I}$ is given in Definition 5.5.

The proof of this statement follows in Sections 6 and 7. In Section 8 we rewrite the summation over $\mathcal{I}$ into a summation over subsets of a quiver associated to $w^{P}$ by [CMP08], see Corollary 8.12. In Section 9 we apply Theorem 5.7 and its reformulation as Corollary 8.12 to all the cominuscule homogeneous spaces, leading to new Laurent polynomial potentials for the cominuscule homogeneous spaces of type $\mathrm{D}_{n}, \mathrm{E}_{6}$ and $\mathrm{E}_{7}$, see Subsections $9.4,9.5$ and 9.6 , respectively.

\section{Proof of the Laurent polynomial expression}

In Section 5 we stated our main result in Theorem 5.7. This section is dedicated to proving this theorem. Before we get started on the proof, let us introduce the following notation:

Definition 6.1. Write $V_{\omega_{i}^{\vee}}$ for the irreducible representation of $\mathfrak{g}^{\vee}$ with highest weight $\omega_{i}^{\vee}$, and denote by $v_{\omega_{i}^{\vee}}^{+}$a choice of a highest weight vector. Denote by $v_{\omega_{i}^{\vee}}^{-}=\bar{w}_{0} \cdot v_{\omega_{i}^{\vee}}^{+}$the associated lowest weight vector. Note that the weight space of $v_{\omega_{i}^{\vee}}^{+}$is one-dimensional, so that the projection of an arbitrary $v \in V_{\omega_{i}^{\vee}}$ to this weight space (parallel to the other weight spaces) is a scalar multiple of $v_{\omega_{i}^{\vee}}^{+}$; we denote this scalar by $\left\langle v, v_{\omega_{i}^{v}}^{+}\right\rangle$.

Remark 6.2. Recall that every representation of a Lie algebra induces a representation of the associated simply-connected Lie group. Here, the highest weight representation $V_{\omega_{i}^{\vee}}$ of $\mathfrak{g}^{\vee}$ induces a representation of the universal cover $\widetilde{G}^{\vee}$ of $G^{\vee}$. Since this representation does not always descend to a representation of $G^{\vee}$, we need to work on $\widetilde{G}^{\vee}$ instead. Because we identified $U_{+}^{\vee}$ and $U_{-}^{\vee}$ with their universal covers, we consider the factors $u_{+}$and $u_{-}$of $z=u_{+} t \bar{w}_{0} u_{-} \in \mathcal{Z}_{P}^{\circ}$ as elements of $\widetilde{G}^{\vee}$. The same holds for the one-parameter subgroups $x_{j}^{\vee}(a) \in U_{+}^{\vee}$ and $y_{j}^{\vee}(a) \in U_{-}^{\vee}$ for $j \in\{1, \ldots, n\}$, which we also consider as elements of $\widetilde{G}^{\vee}$. However, the elements $\dot{s}_{i}$ and $\bar{s}_{i}$ of $G^{\vee}$ associated to $s_{i} \in W$ have multiple lifts to $\widetilde{G}^{\vee}$; we choose the lifts to be the elements obtained by taking the product of the one-parameter subgroups in $\widetilde{G}^{\vee}$, i.e.,

$$
\dot{s}_{i}=x_{i}^{\vee}(1) y_{i}^{\vee}(-1) x_{i}^{\vee}(1) \in \widetilde{G}^{\vee} \quad \text { and } \quad \bar{s}_{i}=x_{i}^{\vee}(-1) y_{i}^{\vee}(1) x_{i}^{\vee}(-1) \in \widetilde{G}^{\vee} .
$$

Note that we abuse notation and denote these lifts in the same way as the original elements. Also note that with these choices we still have $\bar{s}_{i}=\dot{s}_{i}^{-1}$. The elements 
$\dot{w}, \bar{w} \in \widetilde{G}^{\vee}$ associated to $w \in W$ are similarly defined by $\dot{w}=\dot{s}_{i_{1}} \cdots \dot{s}_{i_{j}}$ and $\bar{w}=\bar{s}_{i_{1}} \cdots \bar{s}_{i_{j}}$ respectively, where $w=s_{i_{1}} \cdots s_{i_{j}}$ is a reduced expression. This fixes the lift of $z=u_{+} t \bar{w}_{0} u_{-}$up to a choice of lift of $t \in T^{P} \subset T^{\vee}$; the choices differ by a factor in $\operatorname{ker}\left(\alpha_{k}^{\vee}\right) \subset \widetilde{T}^{\vee}$, so all the lifts have $\alpha_{k}^{\vee}(t)=q$ and we choose one arbitrarily (we will continue to abuse notation and also denote the lift of $t \in T^{P}$ by $\left.t \in \widetilde{T}^{\vee}\right)$.

Remark 6.3. Expressions of the form $\left\langle g \cdot v_{\omega_{i}^{\vee}}^{+}, v_{\omega_{i}^{\vee}}^{+}\right\rangle$are a priori only defined for $g \in \widetilde{G}^{\vee}$, so expressions of that form will always assume the group element $g$ to be elements of the universal cover $\widetilde{G}^{\vee}$. Thus, the abuse of notation in Remark 6.2 should not give rise to ambiguity.

The proof of Theorem 5.7 requires a few intermediate results which we will prove in section 7. Assuming for the moment that these hold, the proof of Theorem 5.7 is a straightforward computation:

Proof of Theorem 5.7. We want to find an expression for

$$
\mathcal{W}_{\mathcal{Z}_{P}^{\circ}}(z)=\mathcal{E}^{*}\left(u_{+}^{-1}\right)+\mathcal{F}^{*}\left(u_{-}\right)=-\mathcal{E}^{*}\left(u_{+}\right)+\mathcal{F}^{*}\left(u_{-}\right)
$$

in terms of the toric coordinates of $z \in \mathcal{Z}_{P}^{\circ}$. First, we calculate $\mathcal{F}^{*}\left(u_{-}\right)$:

Lemma 6.4. For $u_{-} \in U_{-}^{\circ}$ we have $\mathcal{F}^{*}\left(u_{-}\right)=\sum_{i=1}^{\ell} a_{i}$.

Thus, we only need to find the term involving the quantum parameter, which comes from $\mathcal{E}^{*}\left(u_{+}\right)=\sum_{i=1}^{n}\left(e_{i}^{\vee}\right)^{*}\left(u_{+}\right)$. We can rewrite each of the summands of this term as follows:

Lemma 6.5. For $z=u_{+} t \bar{w}_{P} u_{-} \in \mathcal{Z}_{P}^{\circ}$ we have

$$
\left(e_{i}^{\vee}\right)^{*}\left(u_{+}\right)=\alpha_{i}^{\vee}(t) \frac{\left\langle\bar{w}_{0}^{-1} u_{-}^{-1} \bar{w}_{P}^{-1} \bar{s}_{i} \cdot v_{\omega_{i}^{\vee}}^{+}, v_{\omega_{i}^{\vee}}^{+}\right\rangle}{\left\langle\bar{w}_{0}^{-1} u_{-}^{-1} \bar{w}_{P}^{-1} \cdot v_{\omega_{i}^{\vee}}^{+}, v_{\omega_{i}^{\vee}}^{+}\right\rangle} .
$$

It turns out that except for $i=k$, these summands do not contribute to the sum:

Lemma 6.6. For $z=u_{+} t \bar{w}_{P} u_{-} \in \mathcal{Z}_{P}^{\circ}$ and $i \neq k$ (where $k$ is such that $P=P_{k}$ ) we have $\left(e_{i}^{\vee}\right)^{*}\left(u_{+}\right)=0$.

Altogether, we have now found that

$$
\mathcal{E}^{*}\left(u_{+}\right)=\left(e_{k}^{\vee}\right)^{*}\left(u_{+}\right)=q \frac{\left\langle\bar{w}_{0}^{-1} u_{-}^{-1} \bar{w}_{P}^{-1} \bar{s}_{k} \cdot v_{\omega_{k}^{\vee}}^{+}, v_{\omega_{k}^{\vee}}^{+}\right\rangle}{\left\langle\bar{w}_{0}^{-1} u_{-}^{-1} \bar{w}_{P}^{-1} \cdot v_{\omega_{k}^{\vee}}^{+}, v_{\omega_{k}^{\vee}}^{+}\right\rangle},
$$

where $q=\alpha_{k}^{\vee}(t)$. Now we of course need to calculate the denominator and numerator of this quotient:

Lemma 6.7. For $u_{-} \in U_{-}^{\circ}$ we have $\left\langle\bar{w}_{0}^{-1} u_{-}^{-1} \bar{w}_{P}^{-1} \cdot v_{\omega_{k}^{v}}^{+}, v_{\omega_{k}^{\vee}}^{+}\right\rangle=(-1)^{\ell} \prod_{i=1}^{\ell} a_{i}$, where $\ell=\ell\left(w^{P}\right)$. 
Lemma 6.8. For $u_{-} \in U_{-}^{\circ}$ we have

$$
\left\langle\bar{w}_{0}^{-1} u_{-}^{-1} \bar{w}_{P}^{-1} \bar{s}_{k} \cdot v_{\omega_{k}^{\vee}}^{+}, v_{\omega_{k}^{\vee}}^{+}\right\rangle=(-1)^{\ell+1} \sum_{\left(i_{j}\right) \in \mathcal{I}} a_{i_{1}} \cdots a_{i_{\ell^{\prime}}},
$$

where $\mathcal{I}=\left\{\left(i_{1}, \ldots, i_{\ell^{\prime}}\right) \mid 1 \leq i_{1}<i_{2}<\cdots<i_{\ell^{\prime}} \leq \ell\right.$ and $\left.w^{\prime}=s_{r_{i_{1}}} \cdots s_{r_{i_{\ell^{\prime}}}}\right\}$ and where we fixed the reduced expression $w^{P}=s_{r_{1}} \cdots s_{r_{\ell}}$ in Section 2.

Thus, we find

$$
\mathcal{E}^{*}\left(u_{+}\right)=-q \frac{\sum_{\left(i_{j}\right) \in \mathcal{I}} a_{i_{1}} \cdots a_{i_{\ell^{\prime}}}}{\prod_{i=1}^{\ell} a_{i}} .
$$

Inserting this and the expression for $\mathcal{F}^{*}\left(u_{-}\right)$into $\mathcal{W}_{\mathcal{Z}_{P}^{\circ}}(z)=-\mathcal{E}^{*}\left(u_{+}\right)+\mathcal{F}^{*}\left(u_{-}\right)$, we obtain the statement of Theorem 5.7.

\section{Proof of the intermediate results}

We only need to prove the lemmas stated in the proof of Theorem 5.7 to conclude the result.

Remark 7.1. The five intermediate lemmas in the proof of Theorem 5.7 are generalizations of [PR18, Lem. 5.5]. Lemmas 6.4, 6.5 and 6.6 follow similar reasoning in the general case. The proof of Lemma 6.7 requires a modification using the general structure of minuscule representations, while Lemma 6.8 follows a different line of reasoning than its counterpart in [PR18].

The first lemma is very straightforward:

Proof of Lemma 6.4. We want to show that $\mathcal{F}^{*}\left(u_{-}\right)=\sum_{i=1}^{\ell} a_{i}$ for $u_{-} \in U_{-}^{\circ}$.

Recall from equation (6) that $\left(f_{i}^{\vee}\right)^{*}\left(y_{j}(a)\right)=a \delta_{i j}$. From this it follows that

$$
\left(f_{i}^{\vee}\right)^{*}\left(u_{-}\right)=\left(f_{i}^{\vee}\right)^{*}\left(y_{r_{\ell}}^{\vee}\left(a_{\ell}\right) \cdots y_{r_{1}}^{\vee}\left(a_{1}\right)\right)=a_{1} \delta_{i, r_{1}}+\ldots+a_{\ell} \delta_{i, r_{\ell}} .
$$

Summing over all $i \in\{1, \ldots, n\}$, we find $\mathcal{F}^{*}\left(u_{-}\right)=\sum_{i=1}^{n}\left(f_{i}^{\vee}\right)^{*}\left(u_{-}\right)=\sum_{i=1}^{\ell} a_{i}$.

Unfortunately, the other term, $\mathcal{E}^{*}\left(u_{+}\right)=\sum_{i=1}^{n}\left(e_{i}^{\vee}\right)^{*}\left(u_{+}\right)$, will not be as easy. We will first reformulate each of the terms $e_{i}^{\vee}\left(u_{+}\right)$:

Proof of Lemma 6.5. We want to show that

$$
\left(e_{i}^{\vee}\right)^{*}\left(u_{+}\right)=\alpha_{i}^{\vee}(t) \frac{\left\langle\bar{w}_{0}^{-1} u_{-}^{-1} \bar{w}_{P}^{-1} \bar{s}_{i} \cdot v_{\omega_{i}^{\vee}}^{+}, v_{\omega_{i}^{\vee}}^{+}\right\rangle}{\left\langle\bar{w}_{0}^{-1} u_{-}^{-1} \bar{w}_{P}^{-1} \cdot v_{\omega_{i}^{\vee}}^{+}, v_{\omega_{i}^{\vee}}^{+}\right\rangle} .
$$

for $z=u_{+} t \bar{w}_{P} u_{-} \in \mathcal{Z}_{P}^{\circ}$.

First, note that the map $U_{+}^{\vee} \rightarrow \mathbb{C}: u \mapsto\left\langle u \bar{s}_{i} \cdot v_{\omega_{i}^{\vee}}^{+}, v_{\omega_{i}^{\vee}}^{+}\right\rangle$is equal to the unique homomorphism $\left(e_{i}^{\vee}\right)^{*}$ sending $x_{i}^{\vee}(a)=\exp \left(a e_{i}^{\vee}\right)$ to $a$ and the other one-parametersubgroups to zero, so

$$
\left(e_{i}^{\vee}\right)^{*}\left(u_{+}\right)=\left\langle u_{+} \bar{s}_{i} \cdot v_{\omega_{i}^{\vee}}^{+}, v_{\omega_{i}^{\vee}}^{+}\right\rangle .
$$


Next, we use the fact that $u_{+}$is a factor in the decomposition of $z \in \mathcal{Z}_{P}^{\circ}$ as $z=u_{+} t \bar{w}_{P} u_{-}$to find a decomposition for $u_{+}$itself. By definition, we have $z=b_{-} \bar{w}_{0}^{-1}$ for some $b_{-} \in B_{-}^{\vee}$ as $\mathcal{Z}_{P}^{\circ} \subset B_{-}^{\vee} \bar{w}_{0}^{-1}$. In Remark 6.2 we fixed lifts of $z=u_{+} t \bar{w}_{P} u_{-}$and the elements $\dot{s}_{i}$ and $\bar{s}_{i}$ to $\widetilde{G}^{\vee}$. Thus, there is a unique lift of $b_{-} \in B_{-}^{\vee}$, also denoted by $b_{-} \in \widetilde{B}_{-}^{\vee}$, such that $b_{-} \bar{w}_{0}^{-1}=z \in \widetilde{G}^{\vee}$. This gives

$$
u_{+}=b_{-} \bar{w}_{0}^{-1} u_{-}^{-1} \bar{w}_{P}^{-1} t^{-1} \in \widetilde{G}^{\vee} .
$$

Thus, we have to calculate $\left\langle b_{-} \bar{w}_{0}^{-1} u_{-}^{-1} \bar{w}_{P}^{-1} t^{-1} \bar{s}_{i} \cdot v_{\omega_{i}^{\vee}}^{+}, v_{\omega_{i}^{\vee}}^{+}\right\rangle$.

Now, $\bar{s}_{i} \cdot v_{\omega_{i}^{\vee}}^{+}$has weight $\omega_{i}^{\vee}-\alpha_{i}^{\vee}$, so $t^{-1} \in \widetilde{T}^{\vee}$ acts on this vector by scalar multiplication with

$$
\left[\omega_{i}^{\vee}-\alpha_{i}^{\vee}\right]\left(t^{-1}\right)=\frac{\omega_{i}^{\vee}\left(t^{-1}\right)}{\alpha_{i}^{\vee}\left(t^{-1}\right)}=\frac{\alpha_{i}^{\vee}(t)}{\omega_{i}^{\vee}(t)} \in \mathbb{C} .
$$

(Note that weights and roots are written additively.) We conclude

$$
\left(e_{i}^{\vee}\right)^{*}\left(u_{+}\right)=\alpha_{i}^{\vee}(t) \frac{\left\langle b_{-} \bar{w}_{0}^{-1} u_{-}^{-1} \bar{w}_{P}^{-1} \bar{s}_{i} \cdot v_{\omega_{i}^{\vee}}^{+}, v_{\omega_{i}^{\vee}}^{+}\right\rangle}{\omega_{i}^{\vee}(t)} .
$$

Noting that $b_{-} \in \widetilde{B}_{-}^{\vee}$ sends a vector to a linear combination of vectors of equal or lower weight, we see that the only contribution of $b_{-}$to $\left\langle b_{-} \bar{w}_{0}^{-1} u_{-}^{-1} \bar{w}_{P}^{-1} \bar{s}_{i} \cdot v_{\omega_{i}^{\vee}}^{+}, v_{\omega_{i}^{\vee}}^{+}\right\rangle$ will be the factor $\left\langle b_{-} \cdot v_{\omega_{i}^{\vee}}^{+}, v_{\omega_{i}^{\vee}}^{+}\right\rangle$, so we find

$$
\left(e_{i}^{\vee}\right)^{*}\left(u_{+}\right)=\alpha_{i}^{\vee}(t) \frac{\left\langle b_{-} \cdot v_{\omega_{v}^{\vee}}^{+}, v_{\omega_{i}^{\vee}}^{+}\right\rangle}{\omega_{i}^{\vee}(t)}\left\langle\bar{w}_{0}^{-1} u_{-}^{-1} \bar{w}_{P}^{-1} \bar{s}_{i} \cdot v_{\omega_{i}^{\vee}}^{+}, v_{\omega_{i}^{\vee}}^{+}\right\rangle .
$$

Finally, we use the decomposition in (16) in combination with the fact that $\left\langle u_{+} \cdot v_{\omega_{i}^{\vee}}^{+}, v_{\omega_{i}^{\vee}}^{+}\right\rangle=1$ (as $u_{+} \in U_{+}^{\vee}$ ) to conclude that

$$
\begin{aligned}
1 & =\left\langle u_{+} \cdot v_{\omega_{i}^{\vee}}^{+}, v_{\omega_{i}^{\vee}}^{+}\right\rangle=\left\langle b_{-} \bar{w}_{0}^{-1} u_{-}^{-1} \bar{w}_{P}^{-1} t^{-1} \cdot v_{\omega_{i}^{\vee}}^{+}, v_{\omega_{i}^{\vee}}^{+}\right\rangle \\
& =\frac{\left\langle b_{-} \cdot v_{\omega_{i}^{\vee}}^{+}, v_{\omega_{i}^{\vee}}^{+}\right\rangle}{\omega_{i}^{\vee}(t)}\left\langle\bar{w}_{0}^{-1} u_{-}^{-1} \bar{w}_{P}^{-1} \cdot v_{\omega_{i}^{\vee}}^{+}, v_{\omega_{i}^{\vee}}^{+}\right\rangle,
\end{aligned}
$$

where we calculated the contributions of $b_{-}$and $t^{-1}$ in an analogous way as above. Substituting this into (17), we obtain

$$
\left(e_{i}^{\vee}\right)^{*}\left(u_{+}\right)=\alpha_{i}^{\vee}(t) \frac{\left\langle\bar{w}_{0}^{-1} u_{-}^{-1} \bar{w}_{P}^{-1} \bar{s}_{i} \cdot v_{\omega_{i}^{\vee}}^{+}, v_{\omega_{i}^{\vee}}^{+}\right\rangle}{\left\langle\bar{w}_{0}^{-1} u_{-}^{-1} \bar{w}_{P}^{-1} \cdot v_{\omega_{i}^{\vee}}^{+}, v_{\omega_{i}^{\vee}}^{+}\right\rangle},
$$

as in the statement of the lemma.

Lemma 6.6 claims that all of the summands of $\mathcal{E}^{*}\left(u_{+}\right)=\sum_{i=1}^{n}\left(e_{i}^{\vee}\right)^{*}\left(u_{+}\right)$are zero, except for $i=k$ (where $k$ is such that $P=P_{k}$ ). 
Proof of Lemma 6.6. We need to show that $\left(e_{i}^{\vee}\right)^{*}\left(u_{+}\right)=0$ for $z=u_{+} t \bar{w}_{P} u_{-} \in \mathcal{Z}_{P}^{\circ}$ and $i \neq k$ (where $k$ is such that $P=P_{k}$ ).

Considering the expression for $\left(e_{i}^{\vee}\right)^{*}\left(u_{+}\right)$of Lemma 6.5, we need to show that

$$
\left\langle\bar{w}_{0}^{-1} u_{-}^{-1} \bar{w}_{P}^{-1} \bar{s}_{i} \cdot v_{\omega_{i}^{\vee}}^{+}, v_{\omega_{i}^{\vee}}^{+}\right\rangle=0 \quad \text { for } \quad i \neq k .
$$

Recall that we assumed $u_{-} \in U_{-}^{\circ} \subset U_{-}^{P}=U_{-}^{\vee} \cap B_{+}^{\vee} \bar{w}_{P} \bar{w}_{0} B_{+}^{\vee}$ (see Lemma 5.2). Thus, we have $u_{-}^{-1} \in B_{+}^{\vee} \bar{w}_{0}^{-1} \bar{w}_{P}^{-1} B_{+}^{\vee}$; in other words, there are $b_{1}, b_{2} \in B_{+}^{\vee}$ such that $u_{-}^{-1}=b_{1} \bar{w}_{0}^{-1} \bar{w}_{P}^{-1} b_{2}$. Choosing lifts $b_{1}, b_{2} \in \widetilde{B}_{+}^{\vee}$ such that $u_{-}^{-1}=b_{1} \bar{w}_{0}^{-1} \bar{w}_{P}^{-1} b_{2}$ as elements of $\widetilde{G}^{\vee}$ (again abusing notation), it follows that we have to show that

$$
\left\langle\bar{w}_{0}^{-1} b_{1} \bar{w}_{0}^{-1} \bar{w}_{P}^{-1} b_{2} \bar{w}_{P}^{-1} \bar{s}_{i} \cdot v_{\omega_{i}^{\vee}}^{+}, v_{\omega_{i}^{\vee}}^{+}\right\rangle=0 \quad \text { for } \quad i \neq k .
$$

Now, $\bar{w}_{0}^{-1} b_{1} \bar{w}_{0}^{-1} \in \bar{w}_{0}^{-1} \widetilde{B}_{+}^{\vee} \bar{w}_{0}^{-1}=\widetilde{B}_{-}^{\vee}$, so using an analogous argument to the one in the proof of Lemma 6.5, we find that

$\left\langle\bar{w}_{0}^{-1} b_{1} \bar{w}_{0}^{-1} \bar{w}_{P}^{-1} b_{2} \bar{w}_{P}^{-1} \bar{s}_{i} \cdot v_{\omega_{i}^{\vee}}^{+}, v_{\omega_{i}^{\vee}}^{+}\right\rangle=\left\langle\bar{w}_{0}^{-1} b_{1} \bar{w}_{0}^{-1} \cdot v_{\omega_{i}^{\vee}}^{+}, v_{\omega_{i}^{\vee}}^{+}\right\rangle\left\langle\bar{w}_{P}^{-1} b_{2} \bar{w}_{P}^{-1} \bar{s}_{i} \cdot v_{\omega_{i}^{\vee}}^{+}, v_{\omega_{i}^{\vee}}^{+}\right\rangle$.

As $\left\langle\bar{w}_{0}^{-1} b_{1} \bar{w}_{0}^{-1} \cdot v_{\omega_{i}^{\vee}}^{+}, v_{\omega_{i}^{\vee}}^{+}\right\rangle$only contributes a scalar factor, we need to show that

$$
\left\langle\bar{w}_{P}^{-1} b_{2} \bar{w}_{P}^{-1} \bar{s}_{i} \cdot v_{\omega_{i}^{\vee}}^{+}, v_{\omega_{i}^{\vee}}^{+}\right\rangle=0 \quad \text { for } \quad i \neq k .
$$

In other words, it is enough to show that $\bar{w}_{P}^{-1} b_{2} \bar{w}_{P}^{-1} \bar{s}_{i} \cdot v_{\omega_{i}^{\vee}}^{+}$has no components of weight $\omega_{i}^{\vee}$. This is a straightforward argument with weights: $\bar{s}_{i} \cdot v_{\omega_{i}^{\vee}}^{+}$has weight $\omega_{i}^{\vee}-\alpha_{i}^{\vee}$, so that $\bar{w}_{P}^{-1} \bar{s}_{i} \cdot v_{\omega_{i}^{\vee}}^{+}$has weight $w_{P}\left(\omega_{i}^{\vee}-\alpha_{i}^{\vee}\right)$, noting that $w_{P}^{-1}=w_{P}$ as it is the longest element of the Weyl group $W_{P}=\left\langle s_{i} \mid i \neq k\right\rangle$. Now, as $b_{2} \in \widetilde{B}_{+}^{\vee}$, all components of $b_{2} \bar{w}_{P}^{-1} \bar{s}_{i} \cdot v_{\omega_{i}^{\vee}}^{+}$will have weight $w_{P}\left(\omega_{i}^{\vee}-\alpha_{i}^{\vee}\right)+\alpha_{+}^{\vee}$ for some (possibly trivial) sum $\alpha_{+}^{\vee}$ of positive roots. Thus, we find that $\bar{w}_{P}^{-1} b_{2} \bar{w}_{P}^{-1} \bar{s}_{i} \cdot v_{\omega_{i}^{\vee}}^{+}$ has components of weight

$$
w_{P}\left(w_{P}\left(\omega_{i}^{\vee}-\alpha_{i}^{\vee}\right)+\alpha_{+}^{\vee}\right)=\omega_{i}^{\vee}-\alpha_{i}^{\vee}+w_{P}\left(\alpha_{+}^{\vee}\right),
$$

again using $w_{P}^{-1}=w_{P}$. Thus, one of these components has weight $\omega_{i}^{\vee}$ if and only if $w_{P}\left(\alpha_{+}^{\vee}\right)=\alpha_{i}^{\vee}$. However, since $w_{P}$ is the longest element of the Weyl group $W_{P}=\left\langle s_{i} \mid i \neq k\right\rangle$, we know that it maps all the simple roots $\alpha_{i}^{\vee}$ with $i \neq k$ to negative roots, but then we must have that $\alpha_{+}^{\vee}=w_{P}\left(\alpha_{i}^{\vee}\right) \in \Phi_{-}^{\vee}$ is a negative root and definitely not a sum of positive roots, which gives a contradiction. Thus, all components have weight unequal to $\omega_{i}^{\vee}$, implying that (18) holds, which in turn implies the lemma.

Combining Lemmas 6.5 and 6.6, we conclude that

$$
\mathcal{E}^{*}\left(u_{+}\right)=\left(e_{k}^{\vee}\right)^{*}\left(u_{+}\right)=q \frac{\left\langle\bar{w}_{0}^{-1} u_{-}^{-1} \bar{w}_{P}^{-1} \bar{s}_{k} \cdot v_{\omega_{k}^{\vee}}^{+}, v_{\omega_{k}^{\vee}}^{+}\right\rangle}{\left\langle\bar{w}_{0}^{-1} u_{-}^{-1} \bar{w}_{P}^{-1} \cdot v_{\omega_{k}^{\vee}}^{+}, v_{\omega_{k}^{\vee}}^{+}\right\rangle} .
$$

Lemma 6.7 calculates the denominator of this quotient and Lemma 6.8 calculates its numerator: 
Proof of Lemma 6.7. We need to show that for $u_{-} \in U_{-}^{\circ}$ we have

$$
\left\langle\bar{w}_{0}^{-1} u_{-}^{-1} \bar{w}_{P}^{-1} \cdot v_{\omega_{k}^{\vee}}^{+}, v_{\omega_{k}^{\vee}}^{+}\right\rangle=(-1)^{\ell} \prod_{i=1}^{\ell} a_{i}
$$

where $\ell=\ell\left(w^{P}\right)$.

Using Lemma 4.4(iv) we find

$$
\left\langle\bar{w}_{0}^{-1} u_{-}^{-1} \bar{w}_{P}^{-1} \cdot v_{\omega_{k}^{\vee}}^{+}, v_{\omega_{k}^{\vee}}^{+}\right\rangle=\left\langle\bar{w}_{0}^{-1} u_{-}^{-1} \cdot v_{\omega_{k}^{\vee}}^{+}, v_{\omega_{k}^{\vee}}^{+}\right\rangle
$$

By definition of $U_{-}^{\circ}$ (see Definition 5.1), $u_{-}^{-1}$ has a decomposition of the form

$$
u_{-}^{-1}=y_{r_{1}}^{\vee}\left(-a_{1}\right) \cdots y_{r_{\ell}}^{\vee}\left(-a_{\ell}\right),
$$

where the sequence of indices $\left(r_{1}, \ldots, r_{\ell}\right)$ is the same as the one used in the reduced expression $w^{P}=s_{r_{1}} \cdots s_{r_{\ell}}$ fixed in equation (8), see Section 2 . Now, by definition $y_{i}^{\vee}(a)=\exp \left(a f_{i}^{\vee}\right)=1+a f_{i}^{\vee}+\frac{1}{2} a^{2}\left(f_{i}^{\vee}\right)^{2}+\cdots$, but only the first two terms act non-trivially on the representation, since $\left(f_{i}^{\vee}\right)^{2} \cdot v=0$ for all $v \in V_{\omega_{k}^{\vee}}$ according to Theorem 4.2(iv). We conclude that

$$
u_{-}^{-1} \cdot v_{\omega_{k}^{\vee}}^{+}=\left(1-a_{1} f_{r_{1}}^{\vee}\right) \cdots\left(1-a_{\ell} f_{r_{\ell}}^{\vee}\right) \cdot v_{\omega_{k}^{\vee}}^{+}
$$

Note that this is a sum of vectors of different weights, the term of highest weight being $v_{\omega_{k}^{v}}^{+}$(obtained by taking the term with all the identity factors), and the term of lowest weight being (see Lemma 4.4(iv))

$$
\left(-a_{1}\right) \cdots\left(-a_{\ell}\right) f_{r_{1}}^{\vee} \cdots f_{r_{\ell}}^{\vee} \cdot v_{\omega_{k}^{\vee}}^{+}=(-1)^{\ell}\left(\prod_{i=1}^{\ell} a_{i}\right) v_{\omega_{k}^{\vee}}^{-} .
$$

Only the lowest weight term contributes a coefficient to $\left\langle\bar{w}_{0}^{-1} u_{-}^{-1} \cdot v_{\omega_{k}^{\vee}}^{+}, v_{\omega_{k}^{v}}^{+}\right\rangle$as $\bar{w}_{0}^{-1} v_{\omega_{k}^{\vee}}^{-}=v_{\omega_{k}^{\vee}}^{+}$and $\bar{w}_{0}^{-1}$ is a bijection. Thus, we obtain

$$
\left\langle\bar{w}_{0}^{-1} u_{-}^{-1} \bar{w}_{P}^{-1} \cdot v_{\omega_{k}^{\vee}}^{+}, v_{\omega_{k}^{\vee}}^{+}\right\rangle=(-1)^{\ell} \prod_{i=1}^{\ell} a_{i}
$$

as we wanted to show.

Now we turn to the numerator of (19):

Proof of Lemma 6.8. We need to show that for $u_{-} \in U_{-}^{\circ}$ we have

$$
\left\langle\bar{w}_{0}^{-1} u_{-}^{-1} \bar{w}_{P}^{-1} \bar{s}_{k} \cdot v_{\omega_{k}^{\vee}}^{+}, v_{\omega_{k}^{\vee}}^{+}\right\rangle=(-1)^{\ell+1} \sum_{\left(i_{j}\right) \in \mathcal{I}} a_{i_{1}} \cdots a_{i_{\ell^{\prime}}},
$$

where $\mathcal{I}=\left\{\left(i_{1}, \ldots, i_{\ell^{\prime}}\right) \mid 1 \leq i_{1}<i_{2}<\cdots<i_{\ell^{\prime}} \leq \ell\right.$ and $\left.w^{\prime}=s_{r_{i_{1}}} \cdots s_{r_{i_{\ell^{\prime}}}}\right\}$ was defined in Definition 5.5 and where we fixed the reduced expression $w^{P}=s_{r_{1}} \cdots s_{r_{\ell}}$ in equation (8). 
As we saw in the proof of Lemma 6.7, we only need to consider the lowest-weight term of the vector $u_{-}^{-1} \bar{w}_{P}^{-1} \bar{s}_{k} \cdot v_{\omega_{k}^{v}}^{+}$, as it is the only term mapped to $v_{\omega_{k}^{v}}^{+}$by $\bar{w}_{0}^{-1}$. However, $\bar{w}_{P}^{-1} \bar{s}_{k}$ acts non-trivially on $v_{\omega_{k}^{v}}^{+}$, whereas in the proof of Lemma $6.7 \bar{w}_{P}^{-1}$ acted trivially on $v_{\omega_{k}^{v}}^{+}$by Lemma 4.4(iv).

Recall that we had fixed the reduced expression $w_{P}=s_{q_{1}} \cdots s_{q_{m}}$ in equation (8) and that $\bar{s}_{i}^{-1}=\dot{s}_{i}$, so we find that $\bar{w}_{P}^{-1}=\dot{s}_{q_{m}} \cdots \dot{s}_{q_{1}}$. Moreover, note that $\bar{s}_{k} \cdot v_{\omega_{k}^{\vee}}^{+}=f_{k}^{\vee} \cdot v_{\omega_{k}^{\vee}}^{+}=-\dot{s}_{k} \cdot v_{\omega_{k}^{\vee}}^{+}$(see Corollary 4.3). All in all, we find that

$$
\bar{w}_{P}^{-1} \bar{s}_{k} \cdot v_{\omega_{k}^{v}}^{+}=-\dot{s}_{q_{m}} \cdots \dot{s}_{q_{1}} \dot{s}_{k} \cdot v_{\omega_{k}^{v}}^{+} .
$$

In Section 4 we have written $w^{\prime \prime} \in W^{P}$ for the minimal coset representative of the coset $w_{P} s_{k} W_{P}$ (note that $\left.w_{P}^{-1}=w_{P}\right)$ and written $\ell^{\prime \prime}=\ell\left(w^{\prime \prime}\right) \leq \ell\left(w^{P}\right)=\ell$ for its length. Since $s_{q_{m}} \cdots s_{q_{1}}$ is a reduced expression for $w_{P} \in W_{P}$ and $s_{k} \notin W_{P}$, we deduce that $s_{q_{m}} \cdots s_{q_{1}} s_{k}$ is a reduced expression for $w_{P} s_{k}$. Thus, by Lemma 4.4(i) and (ii), we deduce that $\bar{w}_{P}^{-1} \bar{s}_{k} \cdot v_{\omega_{k}^{\vee}}^{+}=-\dot{s}_{q_{m}} \cdots \dot{s}_{q_{1}} \dot{s}_{k} \cdot v_{\omega_{k}}^{+}=-\dot{w}_{P} \dot{s}_{k} \cdot v_{\omega_{k}^{\vee}}^{+}$and that

$$
\bar{w}_{P}^{-1} \bar{s}_{k} \cdot v_{\omega_{k}^{\vee}}^{+}=-\dot{w}_{P} \dot{s}_{k} \cdot v_{\omega_{k}^{\vee}}^{+}=-\dot{w}^{\prime \prime} \cdot v_{\omega_{k}^{\vee}}^{+}=(-1)^{\ell^{\prime \prime}+1} f_{j_{1}}^{\vee} \cdots f_{j_{\ell^{\prime \prime}}}^{\vee} \cdot v_{\omega_{k}^{\vee}}^{+},
$$

where $w^{\prime \prime}=s_{j_{1}} \cdots s_{j_{\ell^{\prime \prime}}}$ is a reduced expression.

Note that in the case $\ell^{\prime \prime}=\ell$ (which only occurs for $\mathbb{C P}^{n}=\operatorname{Gr}(1, n+1)=$ $\left.\mathrm{A}_{n}^{\text {sc }} / P_{1} \cong \mathrm{A}_{n}^{\text {sc }} / P_{n}\right)$ the following arguments become trivial, see Remark 7.3 below.

Next, we need to multiply this vector by $u_{-}^{-1}$, which due to Theorem 4.2(iv) reduces to multiplying by $\left(1-a_{1} f_{r_{1}}^{\vee}\right) \cdots\left(1-a_{\ell} f_{r_{\ell}}^{\vee}\right)$ in the representation. As we mentioned at the start of the proof, we only need to look at the coefficient in front of $v_{\omega_{k}^{\vee}}^{-}$in this product. From Lemma 4.4(iv) we know that $f_{i_{1}}^{\vee} \cdots f_{i_{\ell}}^{\vee} \cdot v_{\omega_{k}^{\vee}}^{+}=$ $v_{\omega_{k}^{\vee}}^{-}$if and only if $s_{i_{1}} \cdots s_{i_{\ell}}=w^{P}$ and this is a reduced expression. So we need exactly those terms of $\left(1-a_{1} f_{r_{1}}^{\vee}\right) \cdots\left(1-a_{\ell} f_{r_{\ell}}^{\vee}\right)$ that complete $f_{j_{1}}^{\vee} \cdots f_{j_{\ell^{\prime \prime}}}^{\vee} \cdot v_{\omega_{k}^{\vee}}^{+}$to $f_{r_{i_{1}}}^{\vee} \cdots f_{r_{i_{\ell-\ell^{\prime \prime}}}}^{\vee} f_{j_{1}}^{\vee} \cdots f_{j_{\ell^{\prime \prime}}}^{\vee} \cdot v_{\omega_{k}^{\vee}}^{+}$in such a way that the indices satisfy the condition $s_{r_{i_{1}}} \cdots s_{r_{i_{\ell-\ell^{\prime \prime}}}} s_{j_{1}} \cdots s_{j_{\ell^{\prime \prime}}}=w^{P}$. However, $s_{j_{1}} \cdots s_{j_{\ell^{\prime \prime}}}=w^{\prime \prime}$ and $s_{r_{1}} \cdots s_{r_{\ell}}=w^{P}$, so we obtain a contributing term for every subexpression $s_{r_{i_{1}}} \cdots s_{r_{i_{\ell-\ell}}}$ of the fixed reduced expression for $w^{P}$ such that $s_{r_{i_{1}}} \cdots s_{r_{i_{\ell-\ell^{\prime \prime}}}} w^{\prime \prime}=w^{P}$. Therefore, every subexpression of $w^{\prime}=w^{P}\left(w^{\prime \prime}\right)^{-1}$ in the fixed reduced expression of $w^{P}$ gives a contributing term.

Now, in Definition 5.5 we defined the set indexing these subexpressions as

$$
\mathcal{I}=\left\{\left(i_{1}, \ldots, i_{\ell^{\prime}}\right) \mid 1 \leq i_{1}<i_{2}<\cdots<i_{\ell^{\prime}} \leq \ell \text { and } w^{\prime}=s_{r_{i_{1}}} \cdots s_{r_{i_{\ell^{\prime}}}}\right\},
$$

where $\ell^{\prime}=\ell\left(w^{\prime}\right)=\ell\left(w^{P}\right)-\ell\left(w^{\prime \prime}\right)=\ell-\ell^{\prime \prime}$. In conclusion, for every $\left(i_{1}, \ldots, i_{\ell}\right) \in \mathcal{I}$, we obtain the following term

$$
-\left(-a_{i_{1}}\right) \cdots\left(-a_{i_{\ell^{\prime}}}\right) f_{r_{i_{1}}}^{\vee} \cdots f_{r_{i_{\ell^{\prime}}}}^{\vee}(-1)^{\ell^{\prime \prime}} f_{j_{1}}^{\vee} \cdots f_{j_{\ell^{\prime \prime}}}^{\vee} \cdot v_{\omega_{k}^{\vee}}^{+}=(-1)^{\ell+1} a_{i_{1}} \cdots a_{i_{\ell^{\prime}}} v_{\omega_{k}^{\vee}}^{-}
$$

and we find that

$$
\left\langle\bar{w}_{0}^{-1} u_{-}^{-1} \bar{w}_{P}^{-1} \bar{s}_{k} \cdot v_{\omega_{k}^{\vee}}^{+}, v_{\omega_{k}^{\vee}}^{+}\right\rangle=(-1)^{\ell+1} \sum_{\left(i_{j}\right) \in \mathcal{I}} a_{i_{1}} \cdots a_{i_{\ell^{\prime}}},
$$

as we wanted to show.

This concludes the last of the intermediate results for the proof of Theorem 5.7. 
Remark 7.2. Note that at no point in the proof of Lemma 6.8 do we fix a reduced expression for $w^{\prime}$, so if $\left(i_{j}\right),\left(i_{j}^{\prime}\right) \in \mathcal{I}$, then we do not necessarily have $r_{i_{j}}=r_{i_{j}^{\prime}}$ for all $j$.

Remark 7.3. Note that if $\ell^{\prime \prime}=\ell$, we have $w^{\prime \prime}=w^{P}$. In this case, equation $(20)$ becomes

$$
-\dot{w}^{\prime \prime} \cdot v_{\omega_{k}^{\vee}}^{+}=-\dot{w}^{P} \cdot v_{\omega_{k}^{\vee}}^{+}=(-1)^{\ell+1} f_{r_{1}}^{\vee} \cdots f_{r_{\ell}}^{\vee} \cdot v_{\omega_{k}^{\vee}}^{+}=(-1)^{\ell+1} v_{\omega_{k}^{\vee}}^{-},
$$

Since $u_{-} \in U_{-}^{\vee}$, we find that $u_{-}^{-1}$ acts trivially on this, so that

$$
\left\langle\bar{w}_{0}^{-1} u_{-}^{-1} \bar{w}_{P}^{-1} \bar{s}_{k} \cdot v_{\omega_{k}^{\vee}}^{+}, v_{\omega_{k}^{\vee}}^{+}\right\rangle=(-1)^{\ell+1}
$$

Of course, $w^{\prime \prime}=w^{P}$ implies that $w^{\prime}=w^{P}\left(w^{\prime \prime}\right)^{-1}=1$. Thus, subexpressions of $w^{\prime}$ inside $w^{P}$ have zero length and there is only one such subexpression so we find $\mathcal{I}=\{\varnothing\}$. We conclude that $\sum_{\left(i_{j}\right) \in \mathcal{I}} \prod_{j=1}^{\ell^{\prime}} a_{r_{i_{j}}}=1$, taking the empty product to be 1. Thus,

$$
\left\langle\bar{w}_{0}^{-1} u_{-}^{-1} \bar{w}_{P}^{-1} \bar{s}_{k} \cdot v_{\omega_{k}^{\vee}}^{+}, v_{\omega_{k}^{\vee}}^{+}\right\rangle=(-1)^{\ell+1} \sum_{\left(i_{j}\right) \in \mathcal{I}} \prod_{j=1}^{\ell^{\prime}} a_{r_{i_{j}}}
$$

also holds in case $\ell^{\prime \prime}=\ell$.

\section{Reformulating the quantum term using quiver subsets}

In the last two sections we proved that Theorem 5.7 gives a local Laurent polynomial expression for the potential constructed by Rietsch in [Rie08]. However, the drawback of the current expression is the effort required to find all the subexpressions of $w^{\prime}$ inside the fixed reduced expression of $w^{P}$. In this section we will use a quiver associated to $w^{P}$ to enumerate all these subexpressions. For this, we need to use the fact that both $w^{P}$ and $w^{\prime}$ are fully commutative:

Definition 8.1. An element $w \in W$ is called fully commutative if every reduced expression of $w$ can be obtained from a given reduced expression by commuting its factors.

Lemma 8.2. Both $w^{P} \in W^{P}$ and $w^{\prime} \in W$ are fully commutative.

Proof. Full commutativity of $w^{P}$ (and in fact of every element of $W^{P}$ ) follows from [Ste96, Thm. 6.1]. Full commutativity of $w^{\prime}$ is now a consequence of the full commutativity of $w^{P}$ due to [Ste96, Prop. 2.4], which states that every element in $W$ obtained from a fully commutative element by removing simple reflections at the right (or left) is fully commutative itself.

In [CMP08] a quiver is associated to $w^{P}$ using the full commutativity property. This quiver is a modification of the quiver introduced in [Per07]. It is defined as follows: 
Definition 8.3 ([CMP08, Def. 2.1]). Given a fixed reduced expression

$$
w^{P}=s_{r_{1}} \cdots s_{r_{\ell}},
$$

e.g., the one fixed in equation (8):

- For $\beta \in\left\{\alpha_{1}, \ldots, \alpha_{n}\right\}$, let $m(\beta)$ be the number of occurrences of $s_{\beta}$ in the reduced expression, i.e., $m(\beta)=\#\left\{j \mid s_{r_{j}}=s_{\beta}\right\}$.

- For $(\beta, j)$ such that $1 \leq j \leq m(\beta)$, let $\mathcal{J}(\beta, j)$ be the index of the $j$ th occurrence of $s_{\beta}$ in the reduced expression (from left to right), i.e., let $\mathcal{J}(\beta, j)$ be the index such that $s_{r_{\mathcal{J}(\beta, j)}}=s_{\beta}$ and such that $\#\left\{\tilde{\jmath} \leq \mathcal{J}(\beta, j) \mid s_{r_{\tilde{\jmath}}}=\right.$ $\left.s_{\beta}\right\}=j$. Also, set $\mathcal{J}(\beta, 0)=0$ and $\mathcal{J}(\beta, m(i)+1)=\infty$.

The quiver is now defined as follows:

- Draw for the $j$ th occurrence of $s_{\beta}$ in the reduced expression for $w^{P}$ a vertex labeled $(\beta, j)$, i.e., the vertices are $(\beta, j)$ for $\beta \in\left\{\alpha_{1}, \ldots, \alpha_{n}\right\}$ and $j \in$ $\{1, \ldots, m(\beta)\}$.

- Draw an arrow from $(\beta, j)$ to $\left(\beta^{\prime}, j^{\prime}\right)$ if $s_{\beta}$ and $s_{\beta^{\prime}}$ do not commute and if the $j^{\prime}$ th occurrence of $s_{\beta^{\prime}}$ is the first one to the right of the $j$ th occurrence of $s_{\beta}$ in the reduced expression for $w^{P}$ and it occurs before the $(j+1)$ th occurrence of $s_{\beta}$, i.e., draw an arrow from $(\beta, j)$ to $\left(\beta^{\prime}, j^{\prime}\right)$ if $\left(s_{\beta} s_{\beta^{\prime}}\right)^{2} \neq 1$ and $\mathcal{J}\left(\beta^{\prime}, j^{\prime}-1\right)<\mathcal{J}(\beta, j)<\mathcal{J}\left(\beta^{\prime}, j^{\prime}\right)<\mathcal{J}(\beta, j+1)$.

We will denote the resulting quiver by $Q_{X}$.

Note that the resulting quiver does not depend on the reduced expression for $w^{P}$ as it is fully commutative; it suffices to check that the quiver is the same after commuting two simple reflections.

Remark 8.4. In Definition 8.3, we associate the quiver $Q_{X}$ to $X=G / P_{k}$. However, as the Laurent polynomial $\mathcal{W}_{\mathcal{Z}_{P}^{\circ}}$ is defined on $\mathcal{Z}_{P}^{\circ} \subset G^{\vee}$, it would be more proper to associate the quiver to the variety $P_{k}^{\vee} \backslash G^{\vee}$ (the left-quotient of $G^{\vee}$ by $P_{k}^{\vee}$ ). This variety has not played a role here, but is closely related to the variety $X^{\vee}$ of equation (9) and Theorem 3.2. In fact, in the articles [MR13], [PR18], [PRW16], [PR13] it is shown that $X^{\vee}$ is isomorphic to a subvariety of $P_{k}^{\vee} \backslash G^{\vee}$ and an expression for the pull-back of $\mathcal{W}: X^{\vee} \times T^{P} \rightarrow \mathbb{C}$ (see Definition 3.5) to $P_{k}^{\vee} \backslash G^{\vee}$ is given. It is expected that such an isomorphic subvariety exists in general. For now, however, we note that $G$ and $G^{\vee}$ have the same Weyl groups $W$ and $W_{P}=\left\langle s_{i} \mid i \neq k\right\rangle \subset W$ as these only depend on the Coxeter diagram underlying the Dynkin diagram of $G^{\vee}$ and the vertex $k$, so that $w^{P}$ and the associated quivers are actually the same. Thus, we will continue to write $Q_{X}$ even though it would be more proper to write $Q_{P_{k}^{\vee} \backslash G^{\vee}}$.

This quiver has as vertices the factors of the reduced expression of $w^{P}$, so every subexpression of $w^{\prime}$ inside the reduced expression will become a subset of vertices of this quiver.

Definition 8.5. We denote the set of subsets of vertices of the quiver $Q_{X}$ that are associated to reduced subexpressions of $w^{\prime}$ inside the reduced expression of $w^{P}$ fixed in Section 2 by $\mathcal{S}$. In other words,

$$
\mathcal{S}=\left\{\left(\left(\beta_{1}, j_{1}\right), \ldots,\left(\beta_{\ell^{\prime}}, j_{\ell^{\prime}}\right)\right) \mid s_{\beta_{1}} \cdots s_{\beta_{\ell^{\prime}}}=w^{\prime}\right\},
$$


where $\ell^{\prime}=\ell\left(w^{\prime}\right)$. Note that we implicitly order the vertices $\left(\beta_{1}, j_{1}\right), \ldots,\left(\beta_{\ell^{\prime}}, j_{\ell^{\prime}}\right)$ such that $\mathcal{J}\left(\beta_{1}, j_{1}\right)<\cdots<\mathcal{J}\left(\beta_{\ell^{\prime}}, j_{\ell^{\prime}}\right)$, but we will still refer to the elements of $\mathcal{S}$ as subsets. ${ }^{3}$

Remark 8.6. Recall from Definition 5.5 that we defined $\mathcal{I}$ as the set of sequences of subindices $\left(i_{1}, \ldots, i_{\ell^{\prime}}\right)$ such that $s_{r_{i_{1}}} \cdots s_{r_{i_{\ell^{\prime}}}}=w^{\prime}$ is a reduced subexpression of $w^{\prime}$ in the fixed reduced expression $w^{P}=s_{r_{1}} \cdots s_{r_{\ell}}$. Note that $\mathcal{J}$ gives rise to a bijection between $\mathcal{S}$ and $\mathcal{I}$, which we will also denote by $\mathcal{J}$ :

$$
\mathcal{J}:\left(\left(\beta_{1}, j_{1}\right), \ldots,\left(\beta_{\ell^{\prime}}, j_{\ell^{\prime}}\right)\right) \mapsto\left(\mathcal{J}\left(\beta_{1}, j_{1}\right), \ldots, \mathcal{J}\left(\beta_{\ell^{\prime}}, j_{\ell^{\prime}}\right)\right) .
$$

This bijection gives the translation between the subsets of vertices of the quiver $Q_{X}$ (in $\mathcal{S}$ ) and their associated reduced subexpressions (in $\mathcal{I}$ ).

Example 8.7. To illustrate the quiver and the subsets associated to the subexpressions, consider the example of the Grassmannian $X=\operatorname{Gr}(4,6)=\mathrm{SL}_{6} / P_{4}$ of type $\mathrm{A}_{5}$. Fixing for $w^{P}$ the reduced expression $w^{P}=\left(s_{2} s_{3} s_{4} s_{5}\right)\left(s_{1} s_{2} s_{3} s_{4}\right)$, we find that $Q_{X}$ is of the form below. Here all edges are arrows are pointing downwards and the $i$ th column of vertices contains the vertices $\left(\alpha_{i}, j\right)$ with $j$ increasing from 1 to $m^{P}\left(\alpha_{i}\right)$ from top to bottom. Above the quiver, we have drawn the labeled Coxeter diagram in such a way that the $i$ th vertex of the diagram is above the $i$ th column of the quiver. The vertex labeled 4 is marked in the Coxeter diagram to signify that we are considering $X=\mathrm{SL}_{6} / P_{4}$, i.e., to signify that $k=4$ in $X=G / P_{k}$. For each vertex $(\beta, j)$, we also give the value of $\mathcal{J}(\beta, j)$.

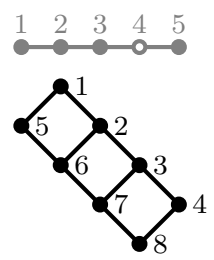

The reduced expression $w^{\prime}=s_{2} s_{3} s_{4}$ is unique and appears as subexpression $\left(s_{2} s_{3} s_{4} s_{5}\right)\left(s_{1} s_{2} s_{3} s_{4}\right),\left(s_{2} s_{3} s_{4} s_{5}\right)\left(s_{1} s_{2} s_{3} s_{4}\right),\left(s_{2} s_{3} s_{4} s_{5}\right)\left(s_{1} s_{2} s_{3} s_{4}\right)$, and finally $\left(s_{2} s_{3} s_{4} s_{5}\right)\left(s_{1} s_{2} s_{3} s_{4}\right)$. These correspond in the quiver $Q_{X}$ to marking the vertices
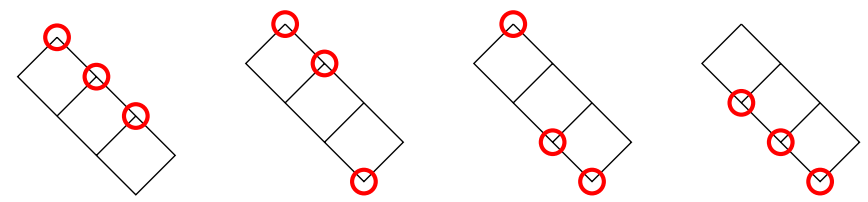

respectively, where we suppressed the vertices.

\footnotetext{
${ }^{3}$ This is to distinguish them from the sequences of subindices that are the elements of $\mathcal{I}$ from Definition 5.5.
} 
It turns out that we can use the quiver $Q_{X}$ to find all the reduced subexpressions of $w^{\prime}$ in $w^{P}$ using two operations that are straightforward when considered as operations on subsets of the quiver. First, we need the following observation:

Remark 8.8. From Definition 8.3, it is clear that we can only have an arrow $(i, j) \rightarrow$ $\left(i^{\prime}, j^{\prime}\right)$ if $\mathcal{J}(i, j)<\mathcal{J}\left(i^{\prime}, j^{\prime}\right)$, so that any path $(i, j) \rightarrow\left(i_{1}, j_{1}\right) \rightarrow \cdots \rightarrow\left(i^{\prime}, j^{\prime}\right)$ between the two vertices corresponds to simple reflections $s_{r_{\mathcal{J}(i, j)}}, s_{r_{\mathcal{J}\left(i_{1}, j_{1}\right)}}, \ldots$, $s_{r_{\mathcal{J}\left(i^{\prime}, j^{\prime}\right)}}$ that appear in that order in the reduced expression of $w^{P}$.

The two operations are given as follows:

Lemma 8.9. Suppose $\left(i_{1}, \ldots, i_{\ell^{\prime}}\right) \in \mathcal{I}$, i.e., suppose it is a sequence of subindices such that $s_{r_{i_{1}}} \cdots s_{r_{i_{\ell^{\prime}}}}=w^{\prime}$ is a reduced subexpression inside the reduced expression $w^{P}=s_{r_{1}} \cdots s_{r_{\ell}}$. Denote by $S \in \mathcal{S}$ the corresponding subset obtained by (the inverse of) the bijection in (21).

(i) For every $\tilde{\imath}$ such that $r_{\tilde{\imath}}=r_{i_{j}}$ and $i_{j-1}<\tilde{\imath}<i_{j+1}$, the sequence of subindices obtained by replacing $i_{j}$ with $\tilde{\imath}$, i.e., $\left(i_{1}, \ldots, i_{j-1}, \tilde{\imath}, i_{j+1}, \ldots, i_{\ell^{\prime}}\right)$, also gives a reduced subexpression of $w^{\prime}$ in $w^{P}$.

In terms of subsets in $\mathcal{S}$, this says that we can replace a vertex $(\beta, j) \in S$ with a vertex $(\beta, \tilde{\jmath})$ if every $\left(\beta^{\prime}, j^{\prime}\right)$ with $\mathcal{J}(\beta, j)<\mathcal{J}\left(\beta^{\prime}, j^{\prime}\right)<\mathcal{J}(\beta, \tilde{\jmath})$ is not an element of $S$ when $j<\tilde{\jmath}\left(\right.$ or $\mathcal{J}(\beta, j)>\mathcal{J}\left(\beta^{\prime}, j^{\prime}\right)>\mathcal{J}(\beta, \tilde{\jmath})$ when $\left.j>\tilde{\jmath}\right)$.

Examples of this operation are the following (using the conventions of Example 8.7):
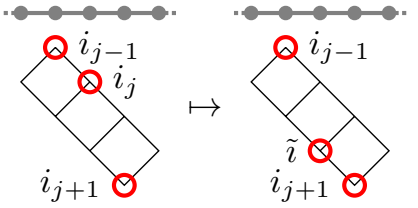
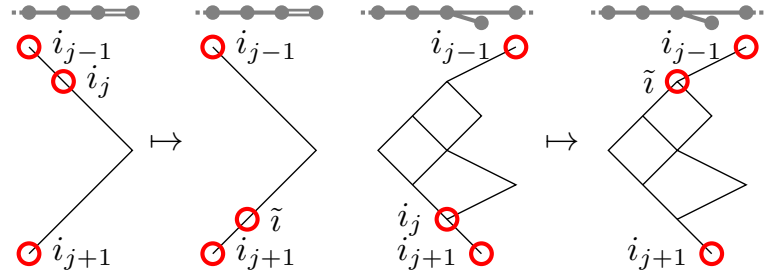

(ii) For every $\tilde{\imath}<i_{j}$ with $r_{\tilde{\imath}}=r_{i_{j}}$ such that there exists a $j^{\prime}$ with $i_{j^{\prime}}<\tilde{\imath}<$ $i_{j^{\prime}+1} \leq i_{j}$ and $\left(s_{r_{i_{j}}} s_{r_{i_{\tilde{j}}}}\right)^{2}=1$ for all $\tilde{\jmath} \in\left\{j^{\prime}+1, \ldots, j-1\right\}$, we have that the sequence of subindices $\left(i_{1}, \ldots, i_{j^{\prime}}, \tilde{\imath}, i_{j^{\prime}+1}, \ldots, i_{j-1}, i_{j+1}, \ldots, i_{\ell^{\prime}}\right)$ is an element of $\mathcal{I}$ as well.

Similarly, for every $\tilde{\imath}>i_{j}$ with $r_{\tilde{\imath}}=r_{i_{j}}$ such that there exists a $j^{\prime}$ with $i_{j} \leq i_{j^{\prime}}<\tilde{\imath}<i_{j^{\prime}+1}$ and $\left(s_{r_{i_{j}}} s_{r_{i_{\tilde{j}}}}\right)^{2}=1$ for all $\tilde{\jmath} \in\left\{j+1, \ldots, j^{\prime}\right\}$, the sequence of subindices $\left(i_{1}, \ldots, i_{j-1}, i_{j+1}, \ldots, i_{j^{\prime}}, \tilde{\imath}, i_{j^{\prime}+1}, \ldots, i_{\ell^{\prime}}\right)$ is also an element of $\mathcal{I}$.

In terms of subsets in $\mathcal{S}$, this says that we can replace a vertex $(\beta, j) \in S$ with a vertex $(\beta, \tilde{\jmath})$ if for every path $(\beta, j) \rightarrow\left(\beta_{1}, j_{1}\right) \rightarrow \ldots \rightarrow(\beta, \tilde{\jmath})$ when $j<\tilde{\jmath}\left(\operatorname{or}(\beta, \tilde{\jmath}) \rightarrow\left(\beta_{1}, j_{1}\right) \rightarrow \ldots \rightarrow(\beta, j)\right.$ when $j>\tilde{\jmath}$ respectively) there is no vertex $\left(\beta^{\prime}, j^{\prime}\right) \in S$ contained in the path such that $\left(s_{\beta} s_{\beta^{\prime}}\right)^{2} \neq 1$. Examples of this operation are: 

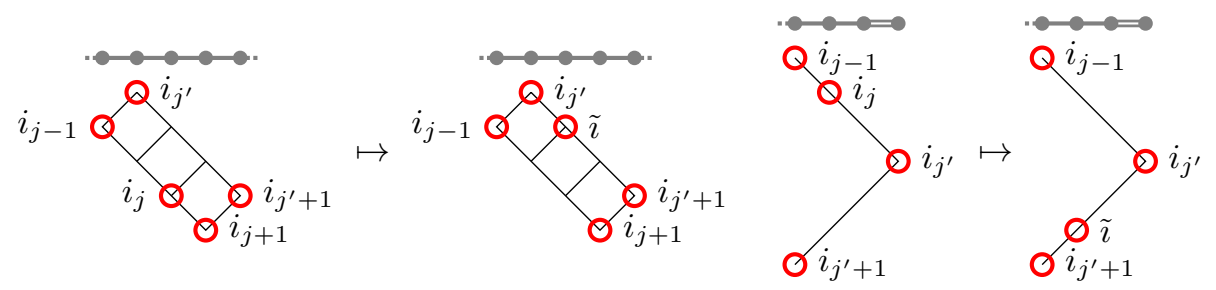

Proof. (i) By assumption, $\left(i_{1}, \ldots, i_{j-1}, \tilde{\imath}, i_{j+1}, \ldots, i_{\ell^{\prime}}\right)$ is an increasing sequence of subindices such that

$$
s_{r_{i_{1}}} \cdots s_{r_{i_{j}-1}} s_{r_{\tilde{\imath}}} s_{r_{i_{j+1}}} \cdots s_{r_{i_{\ell^{\prime}}}}=s_{r_{i_{1}}} \cdots s_{r_{i_{j-1}}} s_{r_{i_{j}}} s_{r_{i_{j+1}}} \cdots s_{r_{i_{\ell^{\prime}}}}=w^{\prime}
$$

and therefore a reduced expression of $w^{\prime}$ in $w^{P}$ as well.

(ii) If $\tilde{\imath}<i_{j}$, we can commute the factor $s_{r_{i_{j}}}$ to the left in the given reduced subexpression to obtain $s_{r_{i_{1}}} \cdots s_{r_{i_{j^{\prime}}}} s_{r_{i_{j}}} s_{r_{i_{j^{\prime}+1}}} \cdots s_{r_{i_{j-1}}} s_{r_{i_{j+1}}} \cdots s_{r_{i_{\ell^{\prime}}}}$ as a reduced expression for $w^{\prime}$. Moreover, this is a subexpression of $w^{\prime}$ in $w^{P}$ since there is a $\tilde{\imath}$ with $i_{j^{\prime}}<\tilde{\imath}<i_{j^{\prime}+1}$ and $r_{\tilde{\imath}}=r_{i_{j}}$, so that $\left(i_{1}, \ldots, i_{j^{\prime}}, \tilde{\imath}, i_{j^{\prime}+1}, \ldots, i_{j-1}, i_{j}, \ldots, i_{\ell^{\prime}}\right)$ is an increasing sequence of subindices.

The case $\tilde{\imath}>i_{j}$ is analogous, except that we commute the factor $s_{r_{i_{j}}}$ to the right.

Remark 8.10. Note that the operation (i) is actually a special case of the operation (ii) where no commutation takes place.

It turns out that these operations suffice to obtain all the reduced subexpressions of $w^{\prime}$ in $w^{P}$. To show this, we introduce a total order on the set of reduced subexpressions by taking the lexicographical order $\prec$ on $\mathcal{I}$. In other words, we have $\left(i_{1}, \ldots, i_{\ell^{\prime}}\right) \prec\left(i_{1}^{\prime}, \ldots, i_{\ell^{\prime}}^{\prime}\right)$ if and only if there exists a $j$ such that $i_{j}<i_{j}^{\prime}$ and $i_{j^{\prime}}=i_{j^{\prime}}^{\prime}$ for $j^{\prime} \in\{1, \ldots, j-1\}$. Let $\left(i_{1}^{*}, \ldots, i_{\ell^{\prime}}^{*}\right)$ be the minimal sequence associated to a reduced subexpression of $w^{\prime}$ in $w^{P}$.

Proposition 8.11. Every reduced subexpression of $w^{\prime}$ in $w^{P}$ can be obtained using the operations of Lemma 8.9 on the reduced subexpression $w^{\prime}=s_{r_{i_{1}^{*}}} \cdots s_{r_{i_{\ell^{\prime}}}}$ that is minimal with respect to the lexicographical order.

Proof. We will show that every non-minimal reduced subexpression can be made smaller using one of the operations in Lemma 8.9. This gives a sequence of operations from any given reduced subexpression to the minimal one. Since it is evident that each operation is invertible, we obtain the statement.

Therefore, let $\left(i_{1}, \ldots, i_{\ell^{\prime}}\right) \in \mathcal{I}$ be non-minimal with respect to the lexicographical order. By definition, there exists a $j$ such that $i_{j}>i_{j}^{*}$ and $i_{j^{\prime}}=i_{j^{\prime}}^{*}$ for all $j^{\prime} \in\{1, \ldots, j-1\}$. We distinguish two cases: $r_{i_{j}}=r_{i_{j}^{*}}$ and $r_{i_{j}} \neq r_{i_{j}^{*}}$.

In the case $r_{i_{j}}=r_{i_{j}^{*}}$, we can apply operation (i) to directly obtain the reduced subexpression $\left(i_{1}, \ldots, i_{j-1}, i_{j}^{*}, i_{j+1}, \ldots, i_{\ell^{\prime}}\right) \prec\left(i_{1}, \ldots, i_{j-1}, i_{j}, i_{j+1}, \ldots, i_{\ell^{\prime}}\right)$. 
Now, consider the case $r_{i_{j}} \neq r_{i_{j}^{*}}$. Suppose that the simple reflection $s_{r_{i_{j}^{*}}}$ occurs for the $N$ th time in the minimal reduced subexpression of $w^{\prime}$ in $w^{P}$, then there must exist some $j^{\prime}>j$ such that $s_{r_{i_{j^{\prime}}}}$ is the $N$ th occurrence of the same simple reflection in the subexpression $\left(i_{1}, \ldots i_{\ell^{\prime}}\right)$. Indeed, $w^{\prime}$ is fully commutative so that each simple reflection appears the same number of times in each reduced expression. (Note that we have $j^{\prime}>j$ since $s_{r_{i_{\tilde{j}}^{*}}}=s_{r_{i_{\tilde{j}}}}$ for $\tilde{\jmath} \in\{1, \ldots, j-1\}$ and $s_{r_{i_{j}}} \neq s_{r_{i_{j}^{*}}}$.) We also know that the simple reflections $s_{r_{i_{\tilde{\jmath}}}}$ commute with $s_{r_{i_{j^{\prime}}}}$ for $\tilde{\jmath} \in\left\{j, \ldots, j^{\prime}-1\right\}$ because of full commutativity of $w^{\prime}$, since these simple reflections have commuted with $s_{r_{i_{j}^{*}}}=s_{r_{i_{j^{\prime}}}}$ going from $\left(i_{1}^{*}, \ldots, i_{\ell^{\prime}}^{*}\right)$ to $\left(i_{1}, \ldots, i_{\ell^{\prime}}\right)$. Thus, we can apply operation (ii) to obtain the reduced subexpression $\left(i_{1}, \ldots, i_{j-1}, i_{j}^{*}, i_{j}, \ldots, i_{j^{\prime}-1}, i_{j^{\prime}+1}, \ldots, i_{\ell^{\prime}}\right) \prec$ $\left(i_{1}, \ldots, i_{\ell^{\prime}}\right)$.

Combining this with Theorem 5.7 and Remark 8.6, we conclude the following:

Corollary 8.12. Let $X=G / P$ be a cominuscule complete homogeneous space with $G$ a simply-connected, simple, complex algebraic group and $P=P_{k}$ a (maximal) parabolic subgroup. The restriction $\mathcal{W}_{\mathcal{Z}_{P}^{\circ}}$ of $\mathcal{W}_{\mathcal{Z}_{P}^{\vee}}$ to $\mathcal{Z}_{P}^{\circ}$ has the following Laurent polynomial expression:

$$
\mathcal{W}_{\mathcal{Z}_{P}^{\circ}}(z)=\sum_{i=1}^{\ell} a_{i}+q \frac{\sum_{S \in \mathcal{S}} \prod_{(\beta, j) \in S} a_{\mathcal{J}(\beta, j)}}{\prod_{i=1}^{\ell} a_{i}} .
$$

Here $z \in \mathcal{Z}_{P}^{\circ}$ is uniquely decomposed as $z=u_{+} t \bar{w}_{P} u_{-}$with $u_{-}=y_{r_{\ell}}^{\vee}\left(a_{\ell}\right) \cdots y_{r_{1}}^{\vee}\left(a_{1}\right)$ in $U_{-}^{\circ}$ as in Corollary 5.4. Also, $q \in \mathbb{C}^{*}$ is given by $q=\alpha_{k}^{\vee}(t)\left(\right.$ with $\left.t \in T^{P}\right)$, and the subindex relabeling $\mathcal{J}$ is defined in Definition 8.3. The set $\mathcal{S}$ is defined in Definition 8.5 and all its elements are obtained using the operations of Lemma 8.9.

\section{Laurent polynomial potentials for all the cominuscule cases}

Theorem 5.7 allows us to calculate Laurent polynomial potentials for the cominuscule homogeneous spaces listed in Table 1, and Corollary 8.12 gives us a tractable way to find all the terms. In this section, we will give reduced expressions for $w^{P}$ and $w^{\prime}$, and describe the quivers $Q_{X}$ for all the cominuscule homogeneous varieties. We refer the reader interested in representative examples of the sets $\mathcal{S}$ and the resulting Laurent polynomial expressions to the arXiv version of this paper [Spa19].

The Laurent polynomials we obtain for quadrics (type $\mathrm{B}_{n}$ and $\mathrm{D}_{n}$ ) and Lagrangian Grassmannians (type $C_{n}$ ) are identical to those found in [PRW16, Props. 2.2 and 3.11] and [PR13, Prop. A.1]. This is to be expected, as Theorem 5.7 is obtained by a generalization of the methods used there.

However, to the best of our knowledge, the Laurent polynomials for orthogonal Grassmannians (type $\mathrm{D}_{n}$ ) for general $n$, the Cayley plane (type $\mathrm{E}_{6}$ ) and the Freudenthal variety (type $E_{7}$ ) have not yet been given. Moreover, all the potentials have a uniform structure resembling Givental's Laurent polynomial potential for projective complete intersections [Giv96], namely they are the sum of the toric coordinates plus a quantum term consisting of a homogeneous polynomial divided by the product of all the toric coordinates. 


\subsection{The Grassmannian}

$X=\operatorname{Gr}(k, n)=\mathrm{SL}_{n} / P_{k}$, considered as a homogeneous space for the special linear group, the simply-connected complex Lie group of type $A_{n-1}$. Note that the parabolic subgroup is given by

$$
P_{k}=\left[\begin{array}{cc}
\mathrm{GL}_{k} & \mathrm{Mat}_{k \times(n-k)} \\
0 & \mathrm{GL}_{n-k}
\end{array}\right] \cap \mathrm{SL}_{n} .
$$

We make two assumptions on $k$ : Firstly, we assume that $k \notin\{1, n-1\}$ : for $k=1$ and $k=n-1$ we find $w^{\prime}=1$. Secondly, we assume without loss of generality that $k>n-k$ : for the remaining cases apply the Dynkin diagram bijection $i \mapsto n-i$. The longest Weyl group element has minimal coset representative

$$
w^{P}=\left(s_{n-k} s_{n+1-k} \cdots s_{n-1}\right)\left(s_{n-1-k} s_{n-k} \cdots s_{n-2}\right) \cdots\left(s_{1} s_{2} \cdots s_{k}\right)
$$

having $n-k$ products in parentheses each with $k$ factors. On the other hand, we find for $w^{\prime}$ the reduced expression

$$
w^{\prime}=\left(s_{n-k} \cdots s_{n-2}\right)\left(s_{n-k-1} \cdots s_{n-3}\right) \cdots\left(s_{2} \cdots s_{k}\right),
$$

having $n-k-1$ products in parentheses each with $k-1$ factors.

The quiver $Q_{X}$ can be written as a $(n-k-1) \times(k-1)$-rectangle and the reduced subexpression for $w^{\prime}$ that is minimal in the lexicographical order is the $(n-k-2) \times(k-2)$-rectangle obtained by removing the bottom row and rightmost column.

Note that there is a clear bijection between the subsets of the quiver $Q_{X}$ and Young diagrams that fit inside a $(n-k-1) \times(k-1)$-rectangle: the unmarked vertices correspond to the contour of the Young diagram. This bijection works in general, so we obtain an alternative description of the quantum term for a general Grassmannian $\operatorname{Gr}(k, n)$ that sums over the Young diagrams that fit inside a $(n-k-1) \times(k-1)$-rectangle.

Remark 9.1. Other Laurent polynomial Landau-Ginzburg models have already been given for Grassmannians. Particularly relevant is the potential given in the paper [EHX97, eqn. (B.25)] (see also [BCFKvS98, Conj. 4.2.2], as well as [MR13, Sect. 6.3]).

The potential $L:\left(\mathbb{C}^{*}\right)^{k(n-k)} \times \mathbb{C}^{*} \rightarrow \mathbb{C}$ of [EHX97] is shown in [MR13], Theorem 4.6, to be a local Laurent polynomial expression for the Landau-Ginzburg model used there. That model is also shown in Proposition 6.7 of [MR13] to be isomorphic to Rietsch's Lie-theoretic Landau-Ginzburg model given in [Rie08], see also Theorem 3.2 here. By construction, the Laurent polynomial potential $\mathcal{W}_{\mathcal{Z}_{P}}$ of Theorem 5.7 and Corollary 8.12 here is a local expression for Rietsch's Lie-theoretic Landau-Ginzburg model. Thus, both $\mathcal{W}_{\mathcal{Z}_{P}^{\circ}}$ and $L$ are local Laurent 
polynomial expressions for the same model. However, it is clear that $\mathcal{W}_{\mathcal{Z}_{P}^{\circ}}$ and $L$ are not isomorphic: the quantum term of $L$ is a Laurent monomial, namely, $T_{[n-k, k]}=q / z_{[n-k, k]}$, whereas the quantum term of $\mathcal{W}_{\mathcal{Z}_{P}^{\circ}}$ is not.

On the other hand, it is straightforward to find a birational map $\Phi$ such that $\Phi^{*} L=\mathcal{W}_{\mathcal{Z}_{P}^{\circ}}$. First, consider the following bijection: for $[i, j] \in \Lambda_{s} \backslash\{[n-k, k]\}$, let $\phi([i, j])=i \cdot k-j+1$, and let $\phi(\varnothing)=(n-k-1) k+1$. Clearly, $\phi$ is a bijection $\Lambda_{s}^{*} \backslash\{[n-k, k], \infty\} \rightarrow\{1, \ldots, k(n-k)\}$. Define $\Phi:\left(\mathbb{C}^{*}\right)^{k(n-k)} \rightarrow\left(\mathbb{C}^{*}\right)^{k(n-k)}$ to be the birational map such that $\Phi^{*}\left(a_{i}\right)=T_{\phi^{-1}(i)}$. By definition, we find (recalling $\left.T_{\infty}=0\right)$

$$
\begin{aligned}
\Phi^{*} \mathcal{W}_{\mathcal{Z}_{P}^{\circ}} & =\Phi^{*}\left(\sum_{i=1}^{k(n-k)} a_{i}+q \frac{P\left(a_{j}\right)}{a_{1} \cdots a_{k(n-k)}}\right) \\
& =\sum_{[i, j] \in \Lambda_{s}^{*} \backslash\{[n-k, k]\}} T_{[i, j]}+q \frac{P\left(T_{\left[i^{\prime}, j^{\prime}\right]}\right)}{T_{\varnothing} T_{[1,1]} T_{[1,2]} \cdots T_{[n-k, k-1]}}
\end{aligned}
$$

where $P$ is the homogeneous polynomial in the numerator of the quantum term of Corollary 8.12. Thus, what remains to be shown is that the quantum term simplifies to $T_{[n-k, k]}=q / z_{[n-k, k]}$, which is a straightforward computation for any given $k$ and $n$.

\subsection{The quadric}

$X=Q_{d}$. Note that both odd- and even-dimensional quadrics are homogeneous for $\operatorname{Spin}_{d+2}$, and that the parabolic subgroup is associated to the first vertex of the Dynkin diagram. Note, however, that $\operatorname{Spin}_{d+2}$ is of a different type depending on whether $d+2=2 n+1$ is odd (type $\mathrm{B}_{n}$ ) or $d+2=2 n$ is even (type $\mathrm{D}_{n}$ ), but the resulting homogeneous spaces are nonetheless similar enough to be considered at the same time. We find for $w^{P}$ the reduced expressions:

$$
w^{P}= \begin{cases}s_{1} s_{2} \cdots s_{n-1}\left(s_{n}\right) s_{n-1} s_{n-2} \cdots s_{1}, & \text { for } d=2 n-1 \\ s_{1} s_{2} \cdots s_{n-2}\left(s_{n-1} s_{n}\right) s_{n-2} s_{n-3} \cdots s_{1}, & \text { for } d=2 n-2\end{cases}
$$

For both odd and even quadrics we find $w^{\prime}=s_{1}$ and in both cases the simple reflection $s_{1}$ only appears as the first and the last factor, so it is easy enough to find the Laurent polynomial potential without using Corollary 8.12. We find the same potential in both cases, namely:

$$
\mathcal{W}_{\mathcal{Z}_{P}^{\circ}}(z)=\sum_{i=1}^{d} a_{i}+q \frac{a_{1}+a_{d}}{\prod_{i=1}^{d} a_{i}}
$$

where $d$ is the dimension of the quadric, and we decomposed $z=u_{+} t \bar{w}_{P} u_{-}$ with $u_{-}=y_{1}^{\vee}\left(-a_{1}\right) y_{2}^{\vee}\left(-a_{2}\right) \cdots y_{1}^{\vee}\left(-a_{d}\right)$ and $q=\alpha_{1}^{\vee}(t)$. Note that this Laurent polynomial expression is indeed identical to the ones obtained in [PRW16, Props. 2.2 and 3.11$]$. 


\subsection{The Lagrangian Grassmannian}

$X=\mathrm{LG}(n, 2 n)=\mathrm{Sp}_{2 n} / P_{n}$, considered as a homogeneous space for the symplectic group of type $C_{n}$. Note that the parabolic subgroup is given by

$$
P_{n}=\left[\begin{array}{cc}
\mathrm{GL}_{n} & \mathrm{Mat}_{n \times n} \\
0 & \mathrm{GL}_{n}
\end{array}\right] \cap \mathrm{Sp}_{2 n} .
$$

The coset representative for the longest Weyl group element has reduced expression:

$$
w^{P}=\left(s_{n}\right)\left(s_{n-1} s_{n}\right)\left(s_{n-2} s_{n-1} s_{n}\right) \cdots\left(s_{1} s_{2} \cdots s_{n}\right),
$$

Similarly, we find for $w^{\prime}$

$$
w^{\prime}=\left(s_{n}\right)\left(s_{n-1} s_{n}\right)\left(s_{n-2} s_{n-1} s_{n}\right) \cdots\left(s_{2} s_{3} \cdots s_{n}\right) .
$$

The quiver $Q_{X}$ can be written as the triangle that is the left half of an $n \times n$ square, and the reduced subexpression for $w^{\prime}$ that is minimal with respect to the lexicographical order is the triangle that is the left half of the $(n-1) \times(n-1)$-square obtained after removing the bottom row and the rightmost column. Note that the Laurent polynomial expression of Theorem 5.7 coincides with the description given in [PR13, Prop. A.1].

Example 9.2. Consider LG(4,8) which is homogeneous for $\mathrm{Sp}_{8}$ of type $\mathrm{C}_{4}$. We find that $w^{P}=\left(s_{4}\right)\left(s_{3} s_{4}\right)\left(s_{2} s_{3} s_{4}\right)\left(s_{1} s_{2} s_{3} s_{4}\right), w^{\prime}=\left(s_{4}\right)\left(s_{3} s_{4}\right)\left(s_{2} s_{3} s_{4}\right)$ and the following quiver:
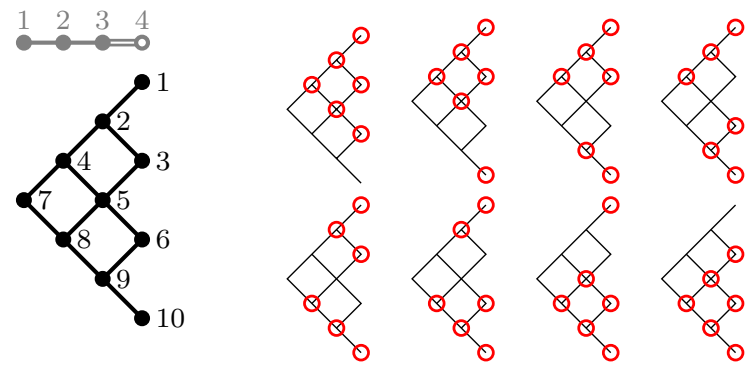

To the right we have drawn the eight subsets corresponding to reduced subexpressions of $w^{\prime}$ inside the reduced expression for $w^{P}$. So, considering the decomposition $z=u_{+} t \bar{w}_{P} u_{-}$with $\left(u_{-}\right)^{-1}=y_{4}^{\vee}\left(-a_{1}\right) y_{3}^{\vee}\left(-a_{2}\right) \cdots y_{4}^{\vee}\left(-a_{10}\right)$ and $q=\alpha_{4}^{\vee}(t)$, we find

$$
\mathcal{W}_{\mathcal{Z}_{P}^{\circ}}(z)=\sum_{i=1}^{10} a_{i}+q \frac{P\left(a_{i}\right)}{a_{1} a_{2} a_{3} a_{4} a_{5} a_{6} a_{7} a_{8} a_{9} a_{10}},
$$

where

$$
\begin{aligned}
P\left(a_{i}\right)= & a_{1} a_{2} a_{3} a_{4} a_{5} a_{6}+a_{1} a_{2} a_{3} a_{4} a_{5} a_{10}+a_{1} a_{2} a_{3} a_{4} a_{9} a_{10}+a_{1} a_{2} a_{4} a_{6} a_{9} a_{10} \\
& +a_{1} a_{2} a_{3} a_{8} a_{9} a_{10}+a_{1} a_{2} a_{6} a_{8} a_{9} a_{10}+a_{1} a_{5} a_{6} a_{8} a_{9} a_{10}+a_{3} a_{5} a_{6} a_{8} a_{9} a_{10}
\end{aligned}
$$

are the eight reduced expression for $w^{\prime}$ obtained from the diagrams above. 


\subsection{The orthogonal Grassmannian}

$X=\mathrm{OG}(n, 2 n)=\operatorname{Spin}_{2 n} / P_{n}$, considered as a homogeneous space for the spin group of type $\mathrm{D}_{n}$. We fix for the minimal coset representative $w^{P}$ of the longest element the reduced expression

$$
w^{P}= \begin{cases}s_{n-1}\left(s_{n-2}\right) s_{n}\left(s_{n-3} s_{n-2}\right) s_{n-1} \cdots\left(s_{1} s_{2} \cdots s_{n-2}\right) s_{n}, & \text { for } n \text { odd } \\ s_{n}\left(s_{n-2}\right) s_{n-1}\left(s_{n-3} s_{n-2}\right) s_{n} \cdots\left(s_{1} s_{2} \cdots s_{n-2}\right) s_{n}, & \text { for } n \text { even. }\end{cases}
$$

Now, for $w^{\prime}$ we find the reduced expression:

$$
w^{\prime}= \begin{cases}s_{n-1}\left(s_{n-2}\right) s_{n}\left(s_{n-3} s_{n-2}\right) s_{n-1} \cdots\left(s_{3} s_{4} \cdots s_{n-2}\right) s_{n}, & \text { for } n \text { odd } \\ s_{n}\left(s_{n-2}\right) s_{n-1}\left(s_{n-3} s_{n-2}\right) s_{n} \cdots\left(s_{3} s_{4} \cdots s_{n-2}\right) s_{n}, & \text { for } n \text { even }\end{cases}
$$

Note that these expressions are very similar to those of Lagrangian Grassmannians except that the expressions for the orthogonal Grassmannians alternate between $s_{n}$ and $s_{n-1}$. The quiver $Q_{X}$ is therefore the left half of an $(n-1) \times(n-1)$-square with the longest column split over two columns. The subset that corresponds to the reduced subexpression for $w^{\prime}$ that is minimal with respect to the lexicographical order is in this case the triangle that is the left half of the $(n-3) \times(n-3)$-square obtained after removing the two bottom rows and the two rightmost columns.

\subsection{The Cayley plane}

$X=\mathbb{O P}^{2}=\mathrm{E}_{6}^{\mathrm{sc}} / P_{6}$, considered as a homogeneous space for the simply-connected Lie group $\mathrm{E}_{6}^{\text {sc }}$ of type $\mathrm{E}_{6}$. We fix for $w^{P}$ the reduced expression

$$
w^{P}=s_{1} s_{3} s_{4} s_{2} s_{5} s_{6} s_{4} s_{5} s_{3} s_{4} s_{2} s_{1} s_{3} s_{4} s_{5} s_{6}
$$

and we find for $w^{\prime}$ the (unique) reduced expression $w^{\prime}=s_{1} s_{3} s_{4} s_{5} s_{6}$. Thus, the quiver is as follows:
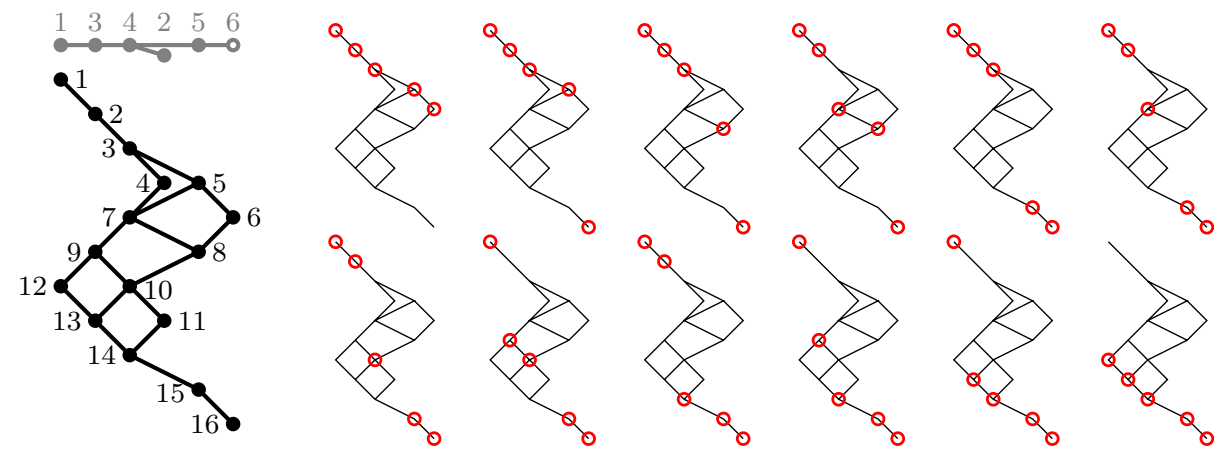

To the right we have drawn the twelve subsets corresponding to subexpressions of $w^{\prime}$. We find for $z=u_{+} t \bar{w}_{P} u_{-}$with $\left(u_{-}\right)^{-1}=y_{1}^{\vee}\left(-a_{1}\right) y_{3}^{\vee}\left(-a_{2}\right) \cdots y_{6}^{\vee}\left(-a_{16}\right)$ 


$$
\mathcal{W}_{\mathcal{Z}_{P}^{\circ}}(z)=\sum_{i=1}^{16} a_{i}+q \frac{P\left(a_{i}\right)}{\prod_{i=1}^{16} a_{i}},
$$

with $q=\alpha_{6}^{\vee}(t)$ and in order of the drawn subsets

$$
\begin{aligned}
P\left(a_{i}\right) & =a_{1} a_{2} a_{3} a_{5} a_{6}+a_{1} a_{2} a_{3} a_{5} a_{16}+a_{1} a_{2} a_{3} a_{8} a_{16}+a_{1} a_{2} a_{7} a_{8} a_{16} \\
& +a_{1} a_{2} a_{3} a_{15} a_{16}+a_{1} a_{2} a_{7} a_{15} a_{16}+a_{1} a_{2} a_{10} a_{15} a_{16}+a_{1} a_{9} a_{10} a_{15} a_{16} \\
& +a_{1} a_{2} a_{14} a_{15} a_{16}+a_{1} a_{9} a_{14} a_{15} a_{16}+a_{1} a_{13} a_{14} a_{15} a_{16}+a_{12} a_{13} a_{14} a_{15} a_{16} .
\end{aligned}
$$

\subsection{The Freudenthal variety}

$X=\mathrm{E}_{7}^{\mathrm{sc}} / P_{7}$, considered as a homogeneous space for the simply-connected Lie group $\mathrm{E}_{7}^{\mathrm{sc}}$ of type $\mathrm{E}_{7}$. We fix for $w^{P}$ the reduced expression

$$
w^{P}=s_{7} s_{6} s_{5} s_{4} s_{2} s_{3} s_{4} s_{5} s_{6} s_{7} s_{1} s_{3} s_{4} s_{2} s_{5} s_{6} s_{4} s_{5} s_{3} s_{4} s_{2} s_{1} s_{3} s_{4} s_{5} s_{6} s_{7},
$$

and $w^{\prime}$ has reduced expression $w^{\prime}=s_{7} s_{6} s_{5} s_{4}\left(s_{2} s_{3}\right) s_{4} s_{5} s_{6} s_{7}$. The quiver $Q_{X}$ is of the form

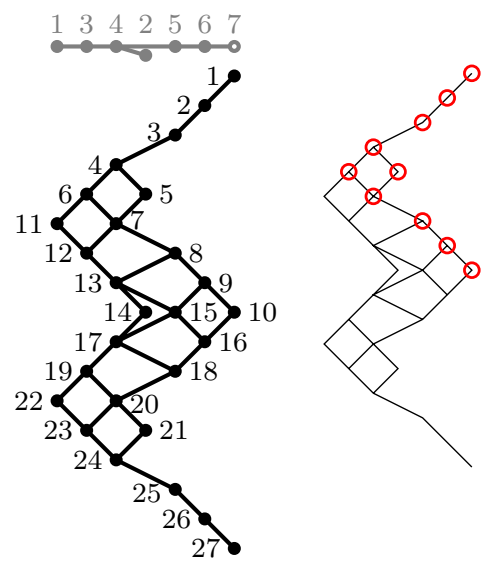

To the right we have drawn the subset corresponding to the reduced subexpression of $w^{\prime}$ that is minimal with respect to the lexicographical order. However, in this case, there are 78 reduced subexpressions, so instead of giving all the corresponding subsets, we will simply list in lexicographical order the elements of $\mathcal{I}$ consisting of sequences of subindices $\left(i_{1}, \ldots, i_{10}\right)$ of $w^{P}=s_{r_{1}} \cdots s_{r_{\ell}}$ such that $w^{\prime}=s_{i_{1}} \cdots s_{i_{10}}$, see Table 2.

We find for $z=u_{+} t \bar{w}_{P} u_{-}$with $\left(u_{-}\right)^{-1}=y_{7}^{\vee}\left(-a_{1}\right) y_{6}^{\vee}\left(-a_{2}\right) \cdots y_{7}^{\vee}\left(-a_{27}\right)$ that

$$
\mathcal{W}_{\mathcal{Z}_{P}^{\circ}}(z)=\sum_{i=1}^{27} a_{i}+q \frac{P\left(a_{i}\right)}{\prod_{i=1}^{27} a_{i}}
$$

with $q=\alpha_{7}^{\vee}(t)$ and $P\left(a_{i}\right)=\sum_{\left(i_{j}\right) \in \mathcal{I}} \prod_{j=1}^{10} a_{i_{j}}$ is a homogeneous polynomial of degree 10 with 78 terms: 
TABLE 2. The full list of all the 78 elements of $\mathcal{I}$ for the Freudenthal variety $\mathrm{E}_{7}^{\mathrm{sc}} / P_{7}$

$\{(1,2,3,4,5,6,7,8,9,10),(1,2,3,4,5,6,7,8,9,27),(1,2,3,4,5,6,7,8,16,27)$, $(1,2,3,4,5,6,7,8,26,27),(1,2,3,4,5,6,7,15,16,27),(1,2,3,4,5,6,7,15,26,27)$, $(1,2,3,4,5,6,7,18,26,27),(1,2,3,4,5,6,7,25,26,27),(1,2,3,4,5,6,13,15,16,27)$, $(1,2,3,4,5,6,13,15,26,27),(1,2,3,4,5,6,13,18,26,27),(1,2,3,4,5,6,13,25,26,27)$, $(1,2,3,4,5,6,17,18,26,27),(1,2,3,4,5,6,17,25,26,27),(1,2,3,4,5,6,20,25,26,27)$, $(1,2,3,4,5,6,24,25,26,27),(1,2,3,4,5,12,13,15,16,27),(1,2,3,4,5,12,13,15,26,27)$, $(1,2,3,4,5,12,13,18,26,27),(1,2,3,4,5,12,13,25,26,27),(1,2,3,4,5,12,17,18,26,27)$, $(1,2,3,4,5,12,17,25,26,27),(1,2,3,4,5,12,20,25,26,27),(1,2,3,4,5,12,24,25,26,27)$, ( $1,2,3,4,5,19,20,25,26,27),(1,2,3,4,5,19,24,25,26,27),(1,2,3,4,5,23,24,25,26,27)$, $(1,2,3,4,6,14,17,18,26,27),(1,2,3,4,6,14,17,25,26,27),(1,2,3,4,6,14,20,25,26,27)$, $(1,2,3,4,6,14,24,25,26,27),(1,2,3,4,6,21,24,25,26,27),(1,2,3,4,12,14,17,18,26,27)$, ( $1,2,3,4,12,14,17,25,26,27),(1,2,3,4,12,14,20,25,26,27),(1,2,3,4,12,14,24,25,26,27)$, $(1,2,3,4,12,21,24,25,26,27),(1,2,3,4,14,19,20,25,26,27),(1,2,3,4,14,19,24,25,26,27)$, ( $1,2,3,4,14,23,24,25,26,27),(1,2,3,4,19,21,24,25,26,27),(1,2,3,4,21,23,24,25,26,27)$, $(1,2,3,7,12,14,17,18,26,27),(1,2,3,7,12,14,17,25,26,27),(1,2,3,7,12,14,20,25,26,27)$, ( $1,2,3,7,12,14,24,25,26,27),(1,2,3,7,12,21,24,25,26,27),(1,2,3,7,14,19,20,25,26,27)$, ( $1,2,3,7,14,19,24,25,26,27),(1,2,3,7,14,23,24,25,26,27),(1,2,3,7,19,21,24,25,26,27)$, ( $1,2,3,7,21,23,24,25,26,27),(1,2,3,13,14,19,20,25,26,27),(1,2,3,13,14,19,24,25,26,27)$, $(1,2,3,13,14,23,24,25,26,27),(1,2,3,13,19,21,24,25,26,27),(1,2,3,13,21,23,24,25,26,27)$, ( $1,2,3,17,19,21,24,25,26,27),(1,2,3,17,21,23,24,25,26,27),(1,2,3,20,21,23,24,25,26,27)$, ( $1,2,8,13,14,19,20,25,26,27),(1,2,8,13,14,19,24,25,26,27),(1,2,8,13,14,23,24,25,26,27)$, $(1,2,8,13,19,21,24,25,26,27),(1,2,8,13,21,23,24,25,26,27),(1,2,8,17,19,21,24,25,26,27)$, ( $1,2,8,17,21,23,24,25,26,27),(1,2,8,20,21,23,24,25,26,27),(1,2,15,17,19,21,24,25,26,27)$, ( $1,2,15,17,21,23,24,25,26,27),(1,2,15,20,21,23,24,25,26,27),(1,2,18,20,21,23,24,25,26,27)$, ( $1,9,15,17,19,21,24,25,26,27),(1,9,15,17,21,23,24,25,26,27),(1,9,15,20,21,23,24,25,26,27)$, $(1,9,18,20,21,23,24,25,26,27),(1,16,18,20,21,23,24,25,26,27),(10,16,18,20,21,23,24,25,26,27)\}$

\section{References}

[BCFKvS98] V. V. Batyrev, I. Ciocan-Fontanine, B. Kim, D. van Straten, Conifold transitions and mirror symmetry for Calabi-Yau complete intersections in Grassmannians, Nuclear Phys. B 514 (1998), no. 3, 640-666.

[Bou68] N. Bourbaki, Groupes et Algèbres de Lie, Éléments de mathématique, Vol. IV-VI, Springer-Verlag, Berlin, 1968.

[CMP08] P. E. Chaput, L. Manivel, N. Perrin, Quantum cohomology of minuscule homogeneous spaces, Transform. Groups 13 (2008), no. 1, 47-89. 
[EHX97] T. Eguchi, K. Hori, C.-S. Xiong, Gravitational quantum cohomology, Internat. J. Modern Phys. A 12 (1997), no. 9, 1743-1782.

[Giv96] A. B. Givental, Equivariant Gromov-Witten invariants, Internat. Math. Res. Notices (1996), no. 13, 613-663.

[Gre08] R. M. Green, Representations of Lie algebras arising from polytopes, Int. Electron. J. Algebra 4 (2008), 27-52.

[Hum75] J. E. Humphreys, Linear Algebraic Groups, Graduate Texts in Mathematics, Vol. 21, Springer-Verlag, New York, 1975.

[Hum78] J. E. Humphreys, Introduction to Lie algebras and Representation Theory, 2nd printing, revised, Graduate Texts in Mathematics, Vol. 9, SpringerVerlag, New York, 1978.

[Lus94] G. Lusztig, Total positivity in reductive groups, in: Lie Theory and Geometry, Progr. Math., Vol. 123, Birkhäuser Boston, Boston, MA, 1994, pp. 531-568.

[MR13] R. Marsh, K. Rietsch, The B-model connection and T-equivariant mirror symmetry for Grassmannians, arXiv:1307.1085 (2013).

[Per07] N. Perrin, Small resolutions of minuscule Schubert varieties, Compos. Math. 143 (2007), no. 5, 1255-1312.

[Pet97] D. Peterson, Quantum cohomology of $G / P$, a lecture course at MIT, Spring Term 1997.

[PR13] C. M. A. Pech, K. Rietsch, A Landau-Ginzburg model for Lagrangian Grassmannians, Langlands duality and relations in quantum cohomology, arXiv:1304.4958 (2013).

[PR18] C. M. A. Pech, K. Rietsch, A comparison of Landau-Ginzburg models for odd dimensional quadrics, Bull. Inst. Math. Acad. Sin. (N.S.) 13 (2018), no. 3, 249-291.

[PRW16] C. M. A. Pech, K. Rietsch, L. Williams, On Landau-Ginzburg models for quadrics and flat sections of Dubrovin connections, Adv. Math. 300 (2016), 275-319.

[Rie08] K. Rietsch, A mirror symmetric construction of $q H_{T}^{*}(G / P)_{(q)}$, Adv. Math. 217 (2008), no. 6, 2401-2442.

[Spa19] P. Spacek, Laurent polynomial Landau-Ginzburg models for cominuscule homogeneous spaces arXiv:1912.09122 (2019).

[Ste96] J. R. Stembridge, On the fully commutative elements of Coxeter groups, J. Algebraic Combin. 5 (1996), no. 4, 353-385.

Open Access This article is licensed under a Creative Commons Attribution 4.0 International License, which permits use, sharing, adaptation, distribution and reproduction in any medium or format, as long as you give appropriate credit to the original author(s) and the source, provide a link to the Creative Commons licence, and indicate if changes were made. The images or other third party material in this article are included in the article's Creative Commons licence, unless indicated 
otherwise in a credit line to the material. If material is not included in the article's Creative Commons licence and your intended use is not permitted by statutory regulation or exceeds the permitted use, you will need to obtain permission directly from the copyright holder. To view a copy of this licence, visit http://creativecommons.org/ licenses/by/4.0/.

Publisher's Note Springer Nature remains neutral with regard to jurisdictional claims in published maps and institutional affiliations. 